\title{
Photoionized emission and absorption features in the high-resolution X-ray spectra of NGC 3783
}

\author{
Junjie $\mathrm{Mao}^{1,2}$, M. Mehdipour ${ }^{1}$, J. S. Kaastra ${ }^{1,3}$, E. Costantini ${ }^{1}$, C. Pinto ${ }^{4}$, G. Branduardi-Raymont ${ }^{5}$, E. Behar ${ }^{6}$, \\ U. Peretz ${ }^{6}$, S. Bianchi ${ }^{7}$, G. A. Kriss ${ }^{8}$, G. Ponti ${ }^{9}$, B. De Marco ${ }^{10}$, P.-O. Petrucci ${ }^{11}$, L. Di Gesu ${ }^{12}$, R. Middei ${ }^{7}$, \\ J. Ebrero ${ }^{13}$, and N. Arav ${ }^{14}$
}

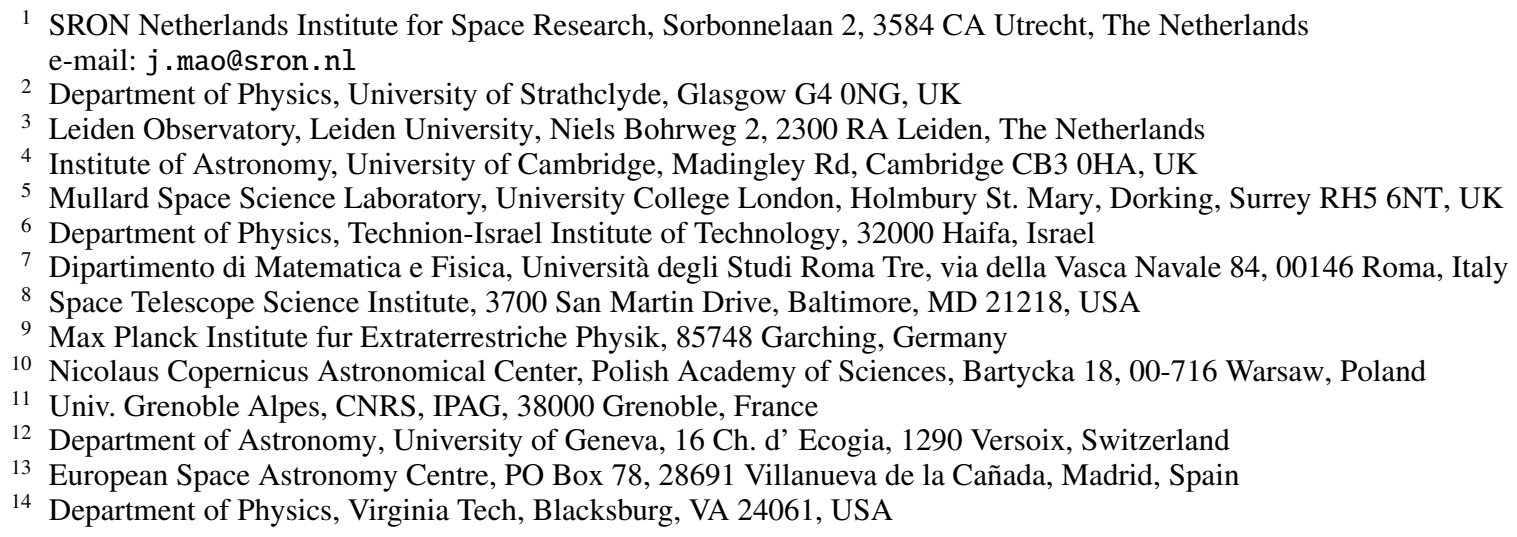

Received 9 April 2018 / Accepted 27 November 2018

\section{ABSTRACT}

\begin{abstract}
Our Swift monitoring program triggered two joint XMM-Newton, NuSTAR, and HST observations on 11 and 21 December 2016 targeting NGC 3783 because its soft X-ray continuum was heavily obscured. Consequently, emission features, including the O vII radiative recombination continuum, stand out above the diminished continuum. We focus on the photoionized emission features in the December 2016 Reflection Grating Spectrometer (RGS) spectra, and compare them to the time-averaged RGS spectrum obtained in 2000-2001 when the continuum was unobscured. A two-phase photoionized plasma is required to account for the narrow emission features. These narrow emission features are weakly varying between 2000-2001 and December 2016. We also find a statistically significant broad emission component in the time-averaged RGS spectrum in 2000-2001. This broad emission component is significantly weaker in December 2016, suggesting that the obscurer is farther away than the X-ray broad-line region. In addition, by analyzing the archival high-resolution X-ray spectra, we find that nine photoionized absorption components with different ionization parameters and kinematics are required for the warm absorber in X-rays.
\end{abstract}

Key words. X-rays: galaxies - galaxies: active - galaxies: Seyfert - galaxies: individual: NGC 3783 - techniques: spectroscopic

\section{Introduction}

The optical spectra of Seyfert 1 galaxies often show broad emission lines with a velocity broadening of a few $10^{3} \mathrm{~km} \mathrm{~s}^{-1}$ and narrow emission lines with a velocity broadening of about a few $10^{2} \mathrm{~km} \mathrm{~s}^{-1}$ (e.g., Blandford et al. 1990). In the soft X-ray band of Seyfert 1 galaxies, broad and narrow emission lines are also observed (e.g., Kaastra et al. 2000; Ogle et al. 2004; Steenbrugge et al. 2009; Longinotti et al. 2010; Costantini et al. 2007, 2016). Although in some Seyfert 2 galaxies the spatial extent of the optical and X-ray narrow emission line regions is remarkably similar (Bianchi et al. 2006), the relation of narrow emission lines in the optical and X-ray bands is not fully understood. Similarly, the connection of optical to X-ray broad emission lines is also poorly understood.

Optical broad emission lines are known to vary because they are dependent on the luminosity of the nucleus (Bentz et al. 2013, and references therein). Optical narrow emission lines (e.g., [O III]) were historically thought to be constant in flux and have been used to calibrate spectroscopic monitoring data (Peterson et al. 2013). However, there is also a growing number of studies (Detmers et al. 2009; Peterson et al. 2013; Denney et al. 2014; Landt et al. 2015) suggesting that the optical and/or X-ray narrow emission lines in active galactic nuclei (AGN, Peterson 1997; Krolik 1999) are variable on long timescales (at least a few years). This is not totally unexpected considering the photoionization origin of these emission lines, the variable ionizing source, and the distance of the narrow-line region (at least a few parsecs, Bennert et al. 2006a,b).

NGC 3783 is a nearby $(z=0.009730$, Theureau et al. 1998) Seyfert 1 galaxy that has been extensively studied for the past few decades in the infrared, optical, UV, and X-ray wavelength ranges (e.g., Kaspi et al. 2000; Kraemer et al. 2001; Onken \& Peterson 2002; Ramírez et al. 2005; Chelouche \& Netzer 2005; Hönig et al. 2013; Goosmann et al. 2016; Fukumura et al. 2018). NGC 3783 has a supermassive 
black hole with $M_{\mathrm{BH}}=(3.0 \pm 0.5) \times 10^{7} M_{\odot}$ (Peterson et al 2004). The radius of the optical broad-line region in NGC 3783 ranges from $0.0012 \mathrm{pc}$ (or 1.4 light-days for $\mathrm{He}$ II) to $0.0086 \mathrm{pc}$ (or 10.2 light-days for $\mathrm{H} \beta$, Peterson et al. 2004).

On 11 and 21 December 2016, our Swift monitoring program $^{1}$ triggered two joint XMM-Newton, NuSTAR, and Hubble Space Telescope (HST) observations targeting NGC 3783 (Mehdipour et al. 2017) because the soft X-ray continuum of NGC 3783 was heavily obscured (see Fig. 2 in Mehdipour et al. 2017). This is similar to the obscuration events discovered in NGC 5548 (Kaastra et al. 2014) and NGC 985 (Ebrero et al. 2016), where strong absorption of the X-ray continuum and outflowing UV broad absorption lines are observed simultaneously. The obscurer in NGC 3783 is found to partially cover the central source with a column density on the order of $10^{27} \mathrm{~m}^{-2}$ (Mehdipour et al. 2017). The obscurer is outflowing with a range of velocities up to $6000 \mathrm{~km} \mathrm{~s}^{-1}$ and it is probably a disk wind at the outer broad-line region of the AGN (Mehdipour et al. 2017). Moreover, based on X-ray data accumulated in the past two decades, Kaastra et al. (2018) suggest that obscuration events with $N_{\mathrm{H}} \gtrsim 10^{26} \mathrm{~m}^{-2}$ are a frequent phenomenon in NGC 3783 . Based on a detailed UV spectral analysis, Kriss et al. (2019) suggest that a collapse of the broad-line region has led to the outburst and triggered the obscuring event. Several other examples of transient X-ray obscuration without simultaneous UV observations have also been observed (e.g., Rivers et al. 2015; Beuchert et al. 2017; Parker et al. 2017; Turner et al. 2018).

Here we present a study of photoionized emission features in the soft X-ray band of NGC 3783 from our recent observations in December 2016 and archival observations in 2000-2001. This paper is structured as follows. In Sect. 2 we list all the observations used. Then we detail the spectral analysis in Sect. 3, including the data treatment and the model description, which is briefly mentioned in Mehdipour et al. (2017). We present the results of our spectral analysis in Sect. 4, as well as discussions of physical implications. Conclusions are available in Sect. 5.

\section{Observations and data reduction}

In 2000-2001 absorption features in NGC 3783 caused by the warm absorber were clearly detectable, as were the $\mathrm{O}$ VIII and $\mathrm{C}_{\mathrm{VI}} \mathrm{Ly} \alpha$ emission lines and the He-like triplets of $\mathrm{O}$ VII (Kaspi et al. 2000; Blustin et al. 2002). However, in December 2016, the soft X-ray continuum was heavily absorbed by the obscurer so that narrow absorption features caused by the warm absorber were hardly visible. Due to this diminished continuum, narrow emission features were better visible in December 2016. This includes $\mathrm{O}$ VII narrow radiative recombination continuum (RRC), which is a characteristic emission feature of a photoionized plasma (Liedahl \& Paerels 1996).

The spectral analysis of such complicated spectra depends crucially on disentangling the true continuum from the effects of the obscuration and absorption features, which may include many lines and edges that are unresolved and overlapping, as well as emission features. That is to say, this requires a model capable of fitting the overall continuum and all the absorption and emission features, including those that cannot be clearly resolved.

Therefore, as described in Mehdipour et al. (2017), we constructed a time-averaged spectrum in 2000 and 2001 to

\footnotetext{
The X-ray hardness variability of eight type-I AGN was monitored with Swift in 2016 in order to find intense obscuration events and thereby study them with joint Hubble Space telescope, XMM-Newton, and NuSTAR observations (Mehdipour et al. 2017).
}

constrain the intrinsic spectral energy distribution (SED) and emission features in the unobscured state. Archival data from the optical monitor (OM, Mason et al. 2001), Reflection Grating Spectrometer (RGS, den Herder et al. 2001), and European Photon Imaging Camera-pn (EPIC-pn, Strüder et al. 2001) from XMM-Newton were used. We also used archival Chandra High-Energy Transmission Grating Spectrometer (HETGS, Canizares et al. 2005) data in 2000, 2001 and 2013 to better constrain the photoionized absorption features, which are rather stable over the 12-year period (Scott et al. 2014). The timeaveraged spectrum has a total exposure of $1.37 \mathrm{Ms}$, with $1.05 \mathrm{Ms}$ for Chandra/HETGS and $324 \mathrm{ks}$ for XMM-Newton/RGS, respectively.

The optical to hard X-ray data in December 2016 were used to determine the intrinsic SED, obscuration effect, and photoionized emission features (present work) in the obscured state. The two data sets in December 2016 were fitted independently as the continua are different (e.g., Fig. 2 in Mehdipour et al. 2017).

All the data used in the present work are listed in Table 1. The exposures of the obscured XMM-Newton/RGS spectra are $110 \mathrm{ks}$ and $56 \mathrm{ks}$, respectively. In addition, we have UV flux from the HST Cosmic Origins Spectrograph (COS, Green et al. 2012). A detailed description of the data reduction for NGC 5548 can be found in Appendix A of Mehdipour et al. (2015), which also applies to the NGC 3783 data used here.

\section{Spectral analysis}

The unobscured (2000-2001) and obscured (December 2016) spectra are constructed with all available data in each epoch as follows:

1. OM flux at V, B, U, UVW1, UVM2, and UVW2 bands;

2. COS flux at $1139 \AA, 1339 \AA, 1477 \AA$, and $1794 \AA$, free from emission and absorption features;

3. first-order RGS data (RGS1 and RGS2 combined) in the 7-37 $\AA$ wavelength range in order to better constrain the emission and absorption features, especially for $\lambda \gtrsim 25 \AA$;

4. first-order medium-energy grating (MEG) data (positive and negative orders combined) in the $6-17 \AA$ wavelength range, which have better energy resolution compared to the RGS data at the same wavelength range. We note that the MEG data are broken into several segments, with the local continuum of each segment re-scaled by $\lesssim \pm 15 \%$ to match the RGS continuum at the same wavelength range. The scaling factor of each segment is 1.158 for $6-7 \AA, 1.120$ for $7-8 \AA, 1.003$ for $8-9 \AA, 1.029$ for $9-11 \AA, 0.889$ for $11-14 \AA$, and 0.850 for $14-17 \AA$. This is to account for observations taken at different epochs, and for the cross-calibration between RGS and MEG;

5. first-order high-energy grating (HEG) data (positive and negative orders combined) in the $1.7-3 \AA$ wavelength range, which has better energy resolution compared to the pn data in the same wavelength range. The HEG data are re-scaled by a factor of 1.218 ;

6. EPIC-pn data in the $1.5-10 \mathrm{keV}$ energy range (i.e., the $1.24-$ $8 \AA$ wavelength range). EPIC-pn data are re-scaled by 1.038 (2000-2001);

7. NUSTAR data in the $10-78 \mathrm{keV}$ energy range. NuSTAR data are re-scaled by 1.013 (11 December 2016) and 1.027 (21 December 2016), respectively.

The above optical to X-ray data are fitted simultaneously, which allows us to constrain the broadband (optical, UV, and X-ray) continuum and the X-ray obscuration, absorption, and emission 
Table 1. NGC 3783 data used for the present spectral analysis.

\begin{tabular}{ccccc}
\hline \hline Observatory & Inst. & ObsID & Start date & Length \\
\hline Chandra & ACIS/HETG & 373 & $2000-01-20$ & $56 \mathrm{ks}$ \\
XMM-Newton & OM, RGS, pn & 0112210101 & $2000-12-28$ & $40 \mathrm{ks}$ \\
Chandra & ACIS/HETG & 2090 & $2001-02-24$ & $166 \mathrm{ks}$ \\
Chandra & ACIS/HETG & 2091 & $2001-02-27$ & $169 \mathrm{ks}$ \\
Chandra & ACIS/HETG & 2092 & $2001-03-10$ & $165 \mathrm{ks}$ \\
Chandra & ACIS/HETG & 2093 & $2001-03-31$ & $166 \mathrm{ks}$ \\
Chandra & ACIS/HETG & 2094 & $2001-06-26$ & $166 \mathrm{ks}$ \\
XMM-Newton & OM, RGS, pn & $0112210201 / 0401$ & $2001-12-17$ & $142 \mathrm{ks}$ \\
XMM-Newton & OM, RGS, pn & $0112210501 / 0601$ & $2001-12-19$ & $142 \mathrm{ks}$ \\
Chandra & ACIS/HETG & 14991 & $2013-03-25$ & $59 \mathrm{ks}$ \\
Chandra & ACIS/HETG & 15626 & $2013-03-27$ & $102 \mathrm{ks}$ \\
\hline HST & COS & LD3E03 & $2016-12-12$ & 2 orbits \\
XMM-Newton & OM, RGS, pn & 0780860901 & $2016-12-11$ & $110 \mathrm{ks}$ \\
NuSTAR & FPMA, FPMB & 80202006002 & $2016-12-11$ & $56 \mathrm{ks}$ \\
\hline HST & COS & LD3E04 & $2016-12-21$ & 2 orbits \\
XMM-Newton & OM, RGS, pn & 0780861001 & $2016-12-21$ & $56 \mathrm{ks}$ \\
NuSTAR & FPMA, FPMB & 80202006004 & $2016-12-21$ & $46 \mathrm{ks}$ \\
\hline
\end{tabular}

features at the same time. We note that the COS grating spectra are analyzed in a separate paper (Kriss et al. 2019).

We used limited but adequate overlapping data to determine the cross-calibration among instruments because the crosscalibration is not a strict constant across the wavelength/energy range. Introducing more overlapping data would introduce more systematic uncertainties, leading to poorer statistics in the end. Nonetheless, we note that we used the best instrument for each wavelength/energy range.

In order to interpret the continuum, absorption, and emission parts of the spectrum at the same time, following Sect. 2.2 of Kaastra et al. (2018), we took the model components listed below into account:

1. The intrinsic broadband spectral energy distribution (SED) of the AGN;

2. The obscuration effect caused by the obscurer and the highionization component in the obscured state;

3. Absorption features caused by the warm absorber;

4. Broad and narrow emission features caused by the X-ray photoionized emitter;

5. Broad and narrow emission lines in the optical/UV;

6. The host galaxy continuum emission in the optical/UV;

7. The Galactic extinction in the optical/UV and absorption in $\mathrm{X}$-rays.

While model components 1,3 , and 4 are described below, we refer readers to Mehdipour et al. (2017) and Kaastra et al. (2018) for details of model components 2, 5, 6, and 7. In Appendix A we discuss the spatial relationships among these components and describe in detail how we combine these components in SPEX. The protosolar abundances of Lodders \& Palme (2009) are used for all plasma models.

\subsection{Intrinsic broadband SED of the AGN}

A photoionization continuum is required for photoionization modeling of the obscurer, warm absorber, and X-ray photoionized emitter. Following a previous analysis of NGC 5548 (Mehdipour et al. 2015), we fitted the optical to X-ray data of NGC 3783 using a model consisting of a Comptonized disk component (COMT, Titarchuk 1994) for optical to soft X-rays, a power-law component (POW), and a neutral reflection component (REFL, Magdziarz \& Zdziarski 1995; Zycki et al. 1999) for hard X-rays. An exponential cutoff is applied to the highand low-energy end of the power-law component (Appendix A). The intrinsic optical to X-ray continuum and all the obscuration, absorption, emission, and extinction effects are fitted simultaneously. Therefore, the fit iterates many times to get the best-fit intrinsic broadband SED of the AGN (results in Sect. 4.1).

\subsection{Photoionization continuum}

A photoionization continuum is required for the photoionization modeling and it can be different from the AGN SED mentioned above. The photoionization continuum received by the obscurer is simply the contemporary AGN SED (Table 2).

For the warm absorber, in 2000-2001, when NGC 3783 is not obscured, its photoionization continuum is also the contemporary AGN SED. However in December 2016 the photoionization continuum for the warm absorber is the contemporary AGN SED with the obscuration effect taken into account.

The photoionization continuum received by the X-ray narrow-line region is assumed to be the 2000-2001 (timeaveraged) AGN SED for all epochs (2000-2001 and December 2016). If the photoionized plasma is too far away from the nucleus and/or the density of the plasma is too low, the plasma is in a quasi-steady state with its ionization balance varying slightly around the mean value corresponding to the mean ionizing flux level over time (Nicastro et al. 1999; Kaastra et al. 2012; Silva et al. 2016). We note that our assumption implies that the obscurer does not subtend a large solid angle so that it barely screens photons from the nucleus to the X-ray narrow emission component or the screened photons have not arrived the X-ray narrow emission component yet.

\subsection{Warm absorber}

We use the photoionization plasma model PION in SPEX to account for the absorption features produced by the warm absorber. PION instantaneously determines the thermal equilibrium, ionization balance, and level populations of all ions from the 


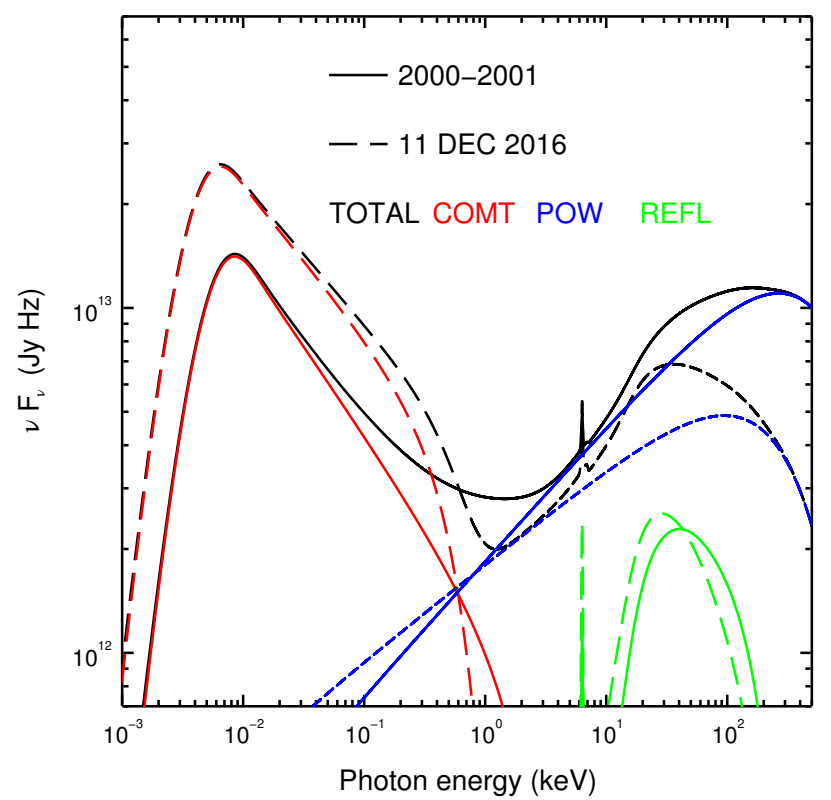

Fig. 1. Intrinsic spectral energy distribution of NGC 3783 for the timeaveraged data in 2000-2001 (solid lines) and 11 December 2016 data (dashed lines). Contributions from individual components are shown in red for the Comptonized disk component (COMT), blue for the powerlaw component (POW), and green for the reflection component (REFL).

input SED and produces an absorption (or emission) model spectrum. As described in Sect. 4.2, we require nine PION absorption components. For the time-averaged unobscured spectrum (2000-2013), we leave hydrogen column densities $\left(N_{\mathrm{H}}\right)$, ionization parameters $(\log \xi)$, and outflow velocities $\left(v_{\text {out }}\right)$ free to vary. After a few trials, microscopic turbulence velocities ${ }^{2}\left(v_{\text {mic }}\right)$ are coupled for components $1-2,3-7$, and 8-9 to reduce unnecessary free parameters. For the obscured spectra (December 2016), all the above parameters are fixed to values obtained in the unobscured spectrum, except the ionization parameters, which are assumed to be proportional to the $1-10^{3}$ Ryd ionizing luminosity. That is to say, the hydrogen number density times distance squared $\left(n_{\mathrm{H}} r^{2}\right)$ of the warm absorber in the obscured state is assumed to be the same as in the unobscured state. In both unobscured and obscured states, each PION absorption component fully covers the line of sight $\left(C_{\mathrm{abs}}=1\right.$, frozen $)$, but has negligible extent $\left(C_{\mathrm{em}}=\Omega / 4 \pi=0\right.$, frozen $)$ with respect to the nucleus.

\subsection{X-ray photoionized emitter}

Similar to the previous analysis on NGC 5548 (Mao et al. 2018), the narrow emission features in NGC 3783 can be reasonably fitted with two PION components (see Sect. 4.3 for details). Whether the soft X-ray continuum is obscured or not, four free parameters of each PION emission component are allowed to vary, namely $N_{\mathrm{H}}, \log \xi, v_{\text {mic }}$, and $C_{\mathrm{em}}$. Since the emission lines are consistent with not outflowing, as shown by previous studies (Kaspi et al. 2002; Behar et al. 2003), the outflow velocity $\left(v_{\text {out }}\right)$ is fixed to zero for each PION emission component. In addition, for each PION emission component, its absorption covering factor $C_{\mathrm{abs}}$ is fixed to zero.

2 The Doppler parameter $b=\left(v_{\mathrm{th}}^{2}+2 v_{\mathrm{mic}}^{2}\right)^{1 / 2}$, where $v_{\mathrm{th}}=\sqrt{2 k T / m}$ is the thermal broadening velocity, $T$ the temperature, and $m$ the atomic mass. The full width at half maximum $F W H M=2 \sqrt{\ln 2} b$. In the PION model in SPEX, $v_{\text {mic }}$ is the "v" parameter.

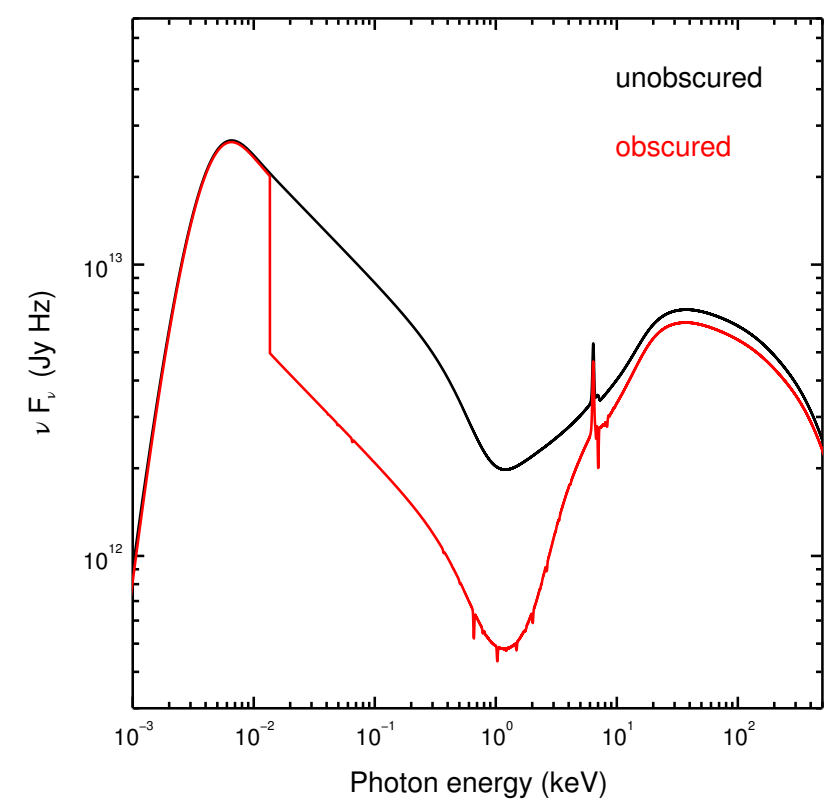

Fig. 2. Intrinsic/unobscured (black) and obscured (red) spectral energy distribution of NGC 3783 on 11 December 2016 (solid lines).

Broad emission features are modeled with a third PION component. For the time-averaged unobscured spectrum (20002001), where the XMM-Newton and Chandra grating spectra are fitted simultaneously, three free parameters of this PION emission component are allowed to vary, including $N_{\mathrm{H}}, \log \xi$, and $C_{\text {em. }}$. For the obscured spectra (December 2016), these parameters are fixed to values obtained in the unobscured spectrum, except the ionization parameters, which are assumed to be proportional to the $1-10^{3}$ Ryd ionizing luminosity (Table 2).

For simplicity, all three emission components are assumed to be free of further absorption by the warm absorber (see discussion in Sect. 4.5).

\section{Results and discussions}

\subsection{Intrinsic broadband SED of the AGN}

The time-averaged intrinsic SED of NGC 3783 in 2000-2013 is shown in Fig. 1. The intrinsic SED on 11 December 2016 is also shown for comparison, which is similar to that on 21 December 2016. Figure 6 of Mehdipour et al. (2017) presents a version with the intrinsic SEDs overplotted with the observational data. The corresponding best-fit continuum parameters are listed in Table 2. Figure 1 shows the comparison of the intrinsic and obscured SED of NGC 3783 on 11 December 2016.

The two observations in December 2016 have similar intrinsic SEDs. However, for the time-averaged SED in 2000-2013, both the Comptonized disk component and the power-law component differ significantly from the December 2016 values.

We should emphasize that we apply a global SED model to fit the optical to hard X-ray data, thus the continuum model in the present work differs from those in previous studies. Kaspi et al. (2001) and Scott et al. (2014) fitted the 1-26 $\AA$ continuum with line-free zones (LFZs). However, as pointed out by Scott et al. (2014), even when LFZs are entirely line-free, absorption edges still contribute to curvature in the spectrum. The $0.5-10 \mathrm{keV}$ continuum of NGC 3783 has also been fitted by a power law with $\Gamma \in(1.5,1.83)$ (Blustin et al. 2002; De Rosa et al. 2002), sometimes including an extra thermal component with $k T \sim 0.1 \mathrm{keV}$ 
Table 2. Best-fit parameters of the intrinsic broadband SED of NGC 3783 in 2000-2001 and December 2016.

\begin{tabular}{lccc}
\hline \hline Date & $2000-2001$ & 11 DEC 2016 & 21 DEC 2016 \\
\hline \multicolumn{4}{c}{ COMT } \\
\hline Norm & $0.95 \pm 0.05$ & $9.7 \pm 0.3$ & $10.3 \pm 0.4$ \\
$T_{\text {seed }}$ & $1.47 \pm 0.05$ & $1.08 \pm 0.03$ & $1.08 \pm 0.02$ \\
$T_{\mathrm{c}}$ & $0.54 \pm 0.02$ & $0.13 \pm 0.02$ & $0.14 \pm 0.03$ \\
$\tau$ & $9.9 \pm 0.2$ & $22.0(\mathrm{f})$ & $22.0(\mathrm{f})$ \\
\hline \multicolumn{4}{c}{ POW } \\
\hline Norm & $2.40 \pm 0.03$ & $2.42 \pm 0.16$ & $3.03 \pm 0.19$ \\
$\Gamma$ & $1.61 \pm 0.01$ & $1.73 \pm 0.02$ & $1.75 \pm 0.02$ \\
$C$-stat / C-expt. & $6092 / 2563$ & $2335 / 1552$ & $2340 / 1555$ \\
$L_{0.3-2 \mathrm{keV}}$ & 1.20 & 1.28 & 1.52 \\
$L_{2-10 \mathrm{keV}}$ & 1.16 & 0.94 & 1.11 \\
$L_{0.001-10 \mathrm{keV}}$ & 10.6 & 18.8 & 21.1 \\
$L_{1-1000 \mathrm{Ryd}}$ & 7.01 & 10.2 & 11.9 \\
\hline
\end{tabular}

Notes. Parameters followed by (f) are fixed in the fit. The temperature of seed photons is in units of $\mathrm{eV}$, the warm corona temperature in $\mathrm{keV}$, the normalization of the warm comptonization (COMT) component in $10^{55} \mathrm{ph} \mathrm{s}^{-1} \mathrm{keV}^{-1}$, and the normalization of the power-law (POW) component in $10^{51} \mathrm{ph} \mathrm{s}^{-1} \mathrm{keV}^{-1}$ at $1 \mathrm{keV}$. The $C$-stat refer to the final best-fit, where all obscuration, absorption, emission and extinction effects are taken into account. The luminosities of COMT plus POW in different energy ranges are in units of $10^{36} \mathrm{~W}$ with uncertainties about $3-5 \%$. All quoted errors refer to the statistical uncertainties at the $68.3 \%$ confidence level.

(Krongold et al. 2003). Fu et al. (2017) constructed a SED with typical AGN SED (Elvis et al. 1994), but scaled with wavelength $\left(\propto \lambda^{-0.27}\right)$ to match the intrinsic UV (1135-1795 $\AA$ ) and X-ray (2-11 $)$ continuum.

\subsection{Warm absorber}

The best-fit ratio of $C$-stat to expected $C$-stat for the timeaveraged spectrum in 2000-2013 is 6092/2563 $\gtrsim 2$ (with 2505 degrees of freedom), which is not statistically acceptable. The poor best-fit ratio is due to the very high photon statistics compared with systematic uncertainties in the instrumental response, the imperfect cross-calibration of different instruments, and the simplicity of our model compared to reality, but the result is still useful to assess whether the observed continuum and all absorption (and emission) features are reasonably accounted for. The best-fit time-averaged (2000, 2001, and 2013) MEG spectrum (6-15 $\AA$ ) is shown in detail in Fig. 3. The best-fit time-averaged (2000-2001) RGS spectrum (8-35 A) was fitted simultaneously, but is shown separately in Fig. 4 for clarity.

There are a total of nine PION absorption components for the warm absorber, with components 1-4 dominating highly ionized ions with large atomic number (e.g., Fe xxVI, Fe xVII, and Si xIV), components 5 and 6 mainly accounting for moderately ionized ions with intermediate atomic number (e.g., Mg IX, O vIII and $\mathrm{Ov}$ ), and components 7-9 for lowly ionized ions with intermediate atomic number (e.g., $\mathrm{N}$ IV and $\mathrm{C}$ IV). Contributions from individual warm absorber components (in percentage) to ions with column density $N_{\text {ion }} \gtrsim 10^{20} \mathrm{~m}^{-2}$ are shown in Fig. 5. Components 7-9 are required in our model because we include the RGS spectrum above $25 \AA$, while previous analyses simply focus on spectra at shorter wavelength ranges with $\lambda \lesssim 25 \AA$ with RGS (Behar et al. 2003) and MEG (Krongold et al. 2003), respectively. Admittedly, the signal-to-noise ratio decreases toward the
Table 3. Best-fit parameters of the warm absorber components in the time-averaged spectra of NGC 3783 in 2000-2013.

\begin{tabular}{ccccc}
\hline \hline Comp. & $\begin{array}{c}N_{\mathrm{H}} \\
10^{25} \mathrm{~m}^{-2}\end{array}$ & $\begin{array}{c}\log _{10}(\xi) \\
10^{-9} \mathrm{~W} \mathrm{~m}\end{array}$ & $\begin{array}{c}v_{\text {mic }} \\
\mathrm{km} \mathrm{s}^{-1}\end{array}$ & $\begin{array}{c}v_{\text {out }} \\
\mathrm{km} \mathrm{s}^{-1}\end{array}$ \\
\hline 1 & $11.1 \pm 0.8$ & $3.02 \pm 0.01$ & $120 \pm 10$ & $-480 \pm 10$ \\
2 & $2.1 \pm 0.2$ & $2.74 \pm 0.03$ & $120(\mathrm{c})$ & $-1300 \pm 25$ \\
3 & $6.1 \pm 0.6$ & $2.55 \pm 0.02$ & $46 \pm 2$ & $-830 \pm 15$ \\
4 & $12.4 \pm 0.5$ & $2.40 \pm 0.01$ & $46(\mathrm{c})$ & $-460 \pm 10$ \\
5 & $5.0 \pm 0.2$ & $1.65 \pm 0.01$ & $46(\mathrm{c})$ & $-575 \pm 10$ \\
6 & $1.2 \pm 0.2$ & $0.92 \pm 0.04$ & $46(\mathrm{c})$ & $-1170 \pm 30$ \\
7 & $0.15_{-0.14}^{+0.05}$ & $0.58_{-0.17}^{+0.28}$ & $46(\mathrm{c})$ & $-1070 \pm 40$ \\
8 & $0.07_{-0.01}^{+0.15}$ & $-0.01_{-0.05}^{+1.82}$ & $790 \pm 100$ & $-1600 \pm 800$ \\
9 & $0.44 \pm 0.03$ & $-0.65 \pm 0.06$ & $790(\mathrm{c})$ & $-1100 \pm 140$ \\
\hline
\end{tabular}

Notes. Microscopic turbulence velocities $\left(v_{\text {mic }}\right)$ followed by (c) are coupled to $v_{\text {mic }}$ of another component with the same value in the fit. Statistical uncertainties are quoted at the $68.3 \%$ confidence level.

longer wavelength, thus the best-fit parameters of components 7-9 are less well constrained. All the best-fit parameters for the warm absorber components of NGC 3783 in the 2000-2013 time-averaged spectra are listed in Table 3.

The hydrogen column density $\left(N_{\mathrm{H}}\right)$ of the warm absorber is dominated by components $1-6$ with $N_{\mathrm{H}}^{\text {total }} \simeq 3.8 \times 10^{26} \mathrm{~m}^{-2}$, similar to previous results by Netzer et al. (2003) with $N_{\mathrm{H}}^{\text {total }} \sim$ $4 \times 10^{26} \mathrm{~m}^{-2}$, which is higher than $N_{\mathrm{H}}^{\text {total }} \sim 2-3 \times 10^{26} \mathrm{~m}^{-2}$ found by Kaspi et al. (2002) and Krongold et al. (2003).

Since the photoionization continuum, abundance table, and number of absorption components used by different authors are different, we do not compare the ionization parameters with previous results (e.g., Kaspi et al. 2002; Netzer et al. 2003; Krongold et al. 2003).

In the present work, the microscopic turbulence velocities of the absorption components are $50 \mathrm{~km} \mathrm{~s}^{-1}$ for Components $3-7,120 \mathrm{~km} \mathrm{~s}^{-1}$ for Components $1-2$, and $800 \mathrm{~km} \mathrm{~s}^{-1}$ for Components 8 and 9 (Table 3 ). The microscopic turbulence velocity found in the literature also covers a wide range of values, with $\sim 100 \mathrm{~km} \mathrm{~s}^{-1}$ for Kaspi et al. (2000) and Behar et al. (2003), $\sim 200 \mathrm{~km} \mathrm{~s}^{-1}$ for Krongold et al. (2003) and Netzer et al. (2003), and up to $400-600 \mathrm{~km} \mathrm{~s}^{-1}$ for a few ions in Table 3 of Kaspi et al. (2002).

Our best-fit outflow velocities vary for different absorption components, ranging from $-450 \mathrm{~km} \mathrm{~s}^{-1}$ to $-1300 \mathrm{~km} \mathrm{~s}^{-1}$. A wide range of outflow velocities are also reported in the literature, including -300 to $-1000 \mathrm{~km} \mathrm{~s}^{-1}$ by Kaspi et al. (2002), -470 to $-800 \mathrm{~km} \mathrm{~s}^{-1}$ by Behar et al. (2003), $-750 \mathrm{~km} \mathrm{~s}^{-1}$ by Krongold et al. (2003), and -400 to $-1300 \mathrm{~km} \mathrm{~s}^{-1}$ by Netzer et al. (2003).

We also note that the PION model in the SPEX code takes advantage of recently updated atomic data, which is more accurate and complete than previous models by Krongold et al. (2003; K03) and Netzer et al. (2003; N03). The previous models were able to fit some of the strong absorption lines, but not enough to fit the global spectrum. As pointed out by Netzer et al. (2003), the K03 model provides a better fit to the Fe UTA (unresolved transition array), yet fails to fit the Six and Si xI lines around $6.8 \AA$. The N03 model has a better fit over the 5-7 wavelength range while the UTA features are poorly fitted. As for the PION model, we refer readers to Sect. 4.29 of the SPEX manual for a detailed description of the model, Mehdipour et al. (2016) for a comparison with other popular photoionization 

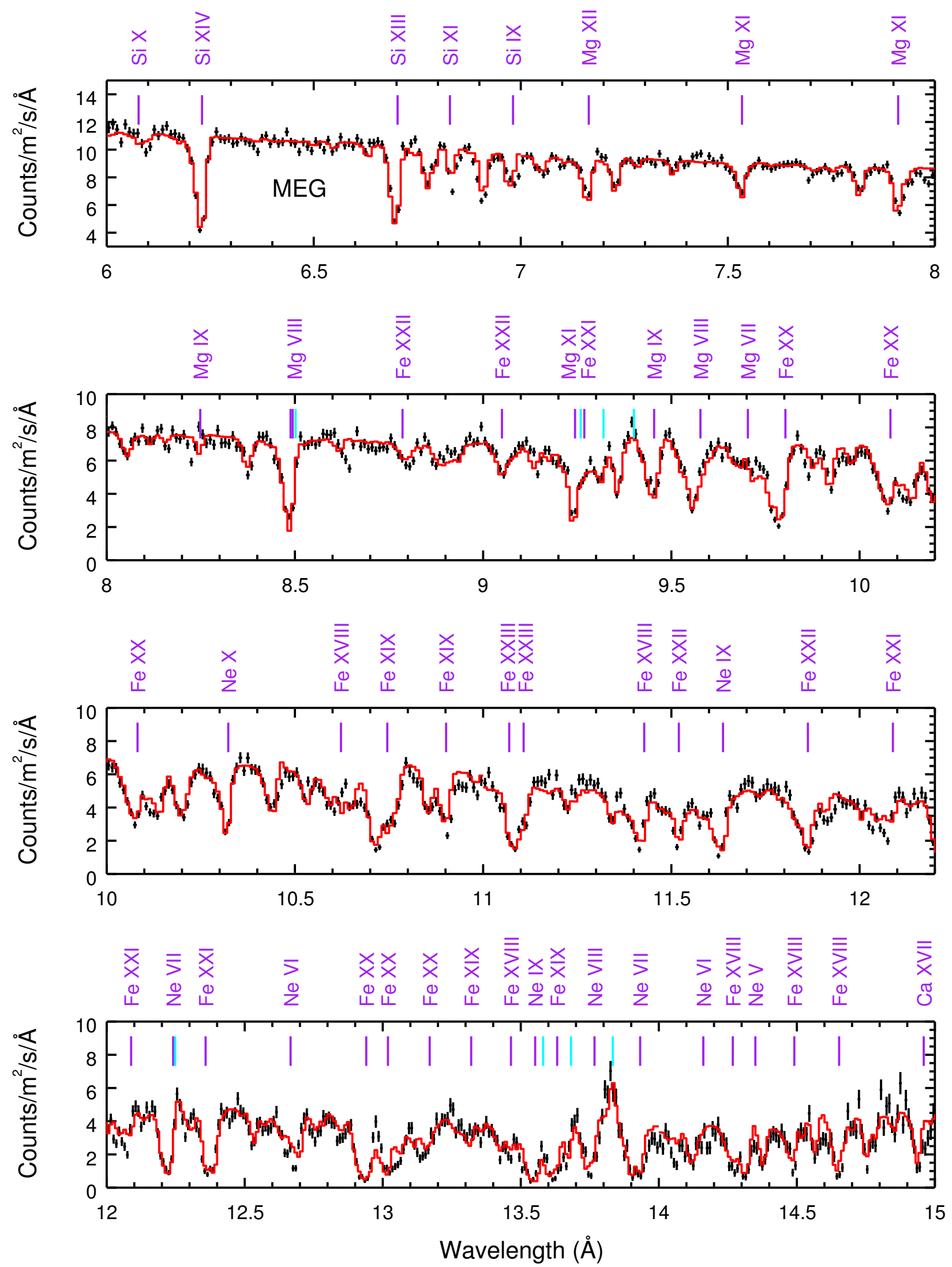

Fig. 3. Best fit to the time-averaged MEG spectrum (in the observed frame) of NGC 3783 observed in 2000, 2001, and 2013. Most of the prominent absorption and emission features are labeled. The solid vertical lines in purple (blue) indicate the photoionized absorption (emission) features. 

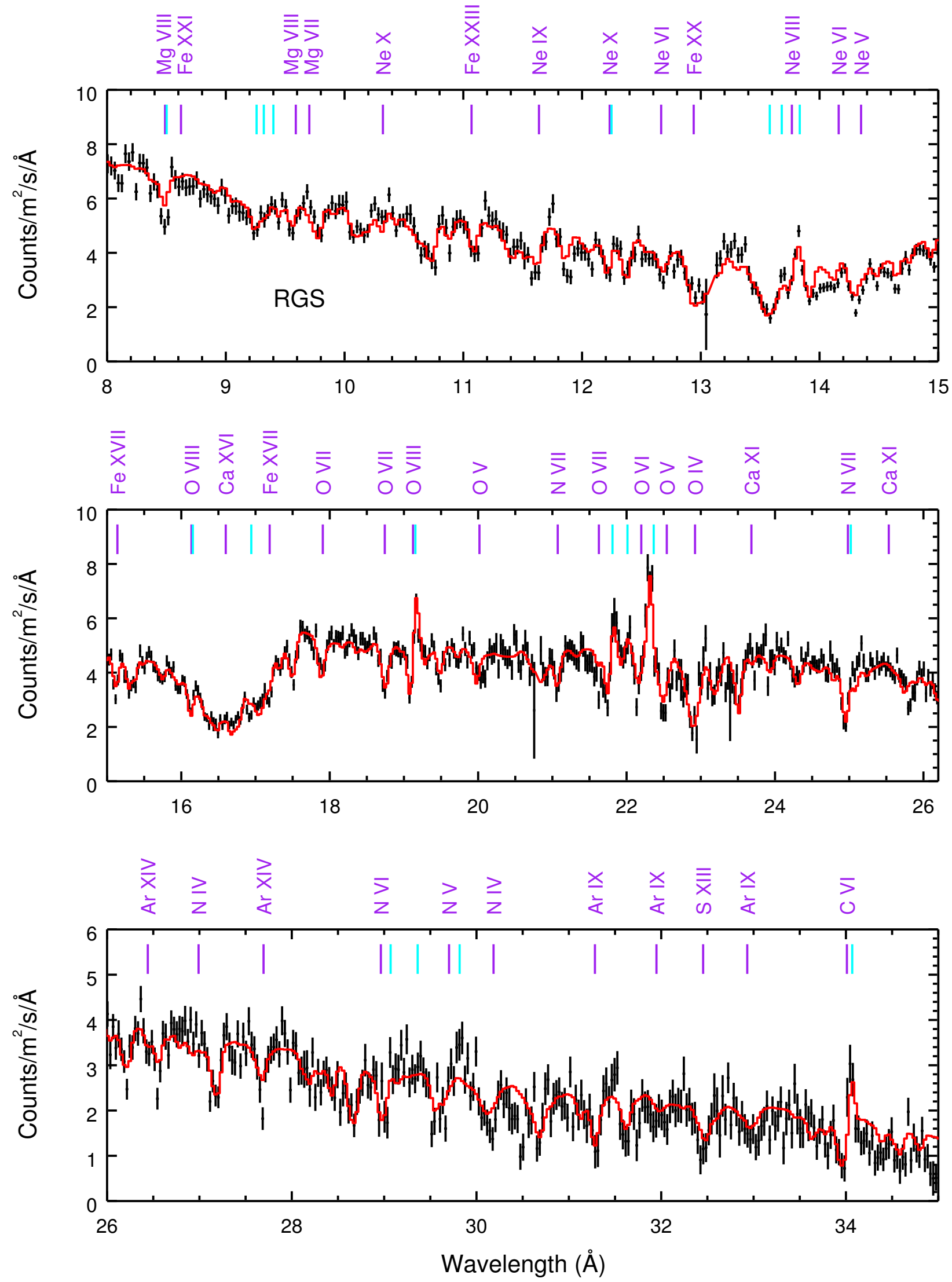

Fig. 4. Similar to Fig. 3, but for the time-averaged RGS spectrum (in the observed frame) of NGC 3783 observed in 2000 and 2001. 


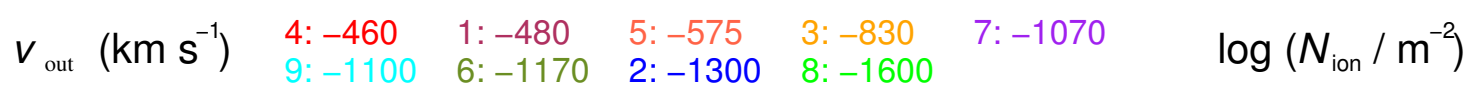

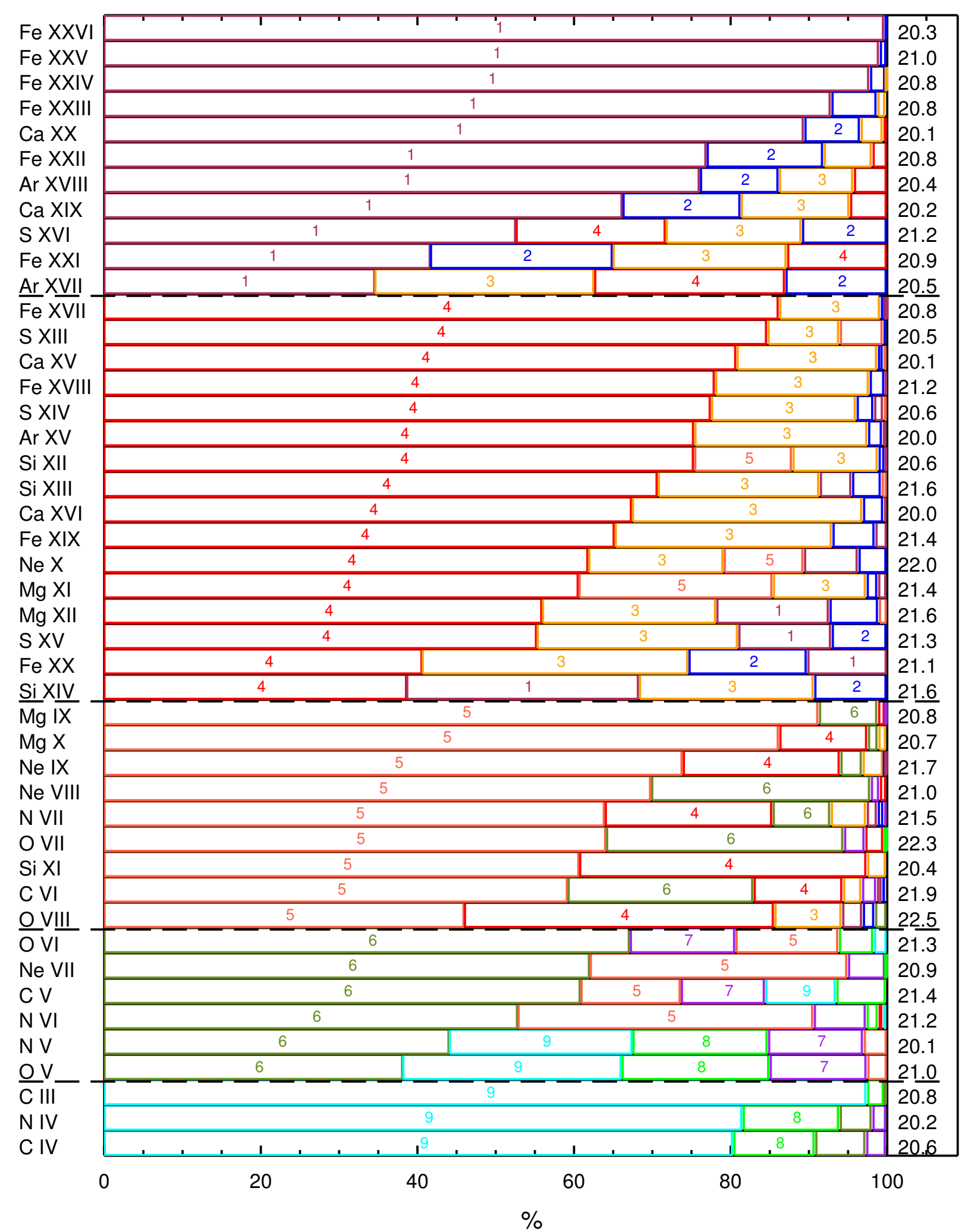

Fig. 5. Ionic column density (units: $\mathrm{m}^{-2}$, in log-scale listed to the right) with contributions from individual warm absorber components (in percentage). Ions with $N_{\text {ion }} \gtrsim 10^{20} \mathrm{~m}^{-2}$ are listed to the left. The warm absorber components are color-coded with outflow velocities ( $\left.v_{\text {out }}\right)$ listed to the top. The ionization parameters of the warm absorber components increase from component 9 to component 1. 

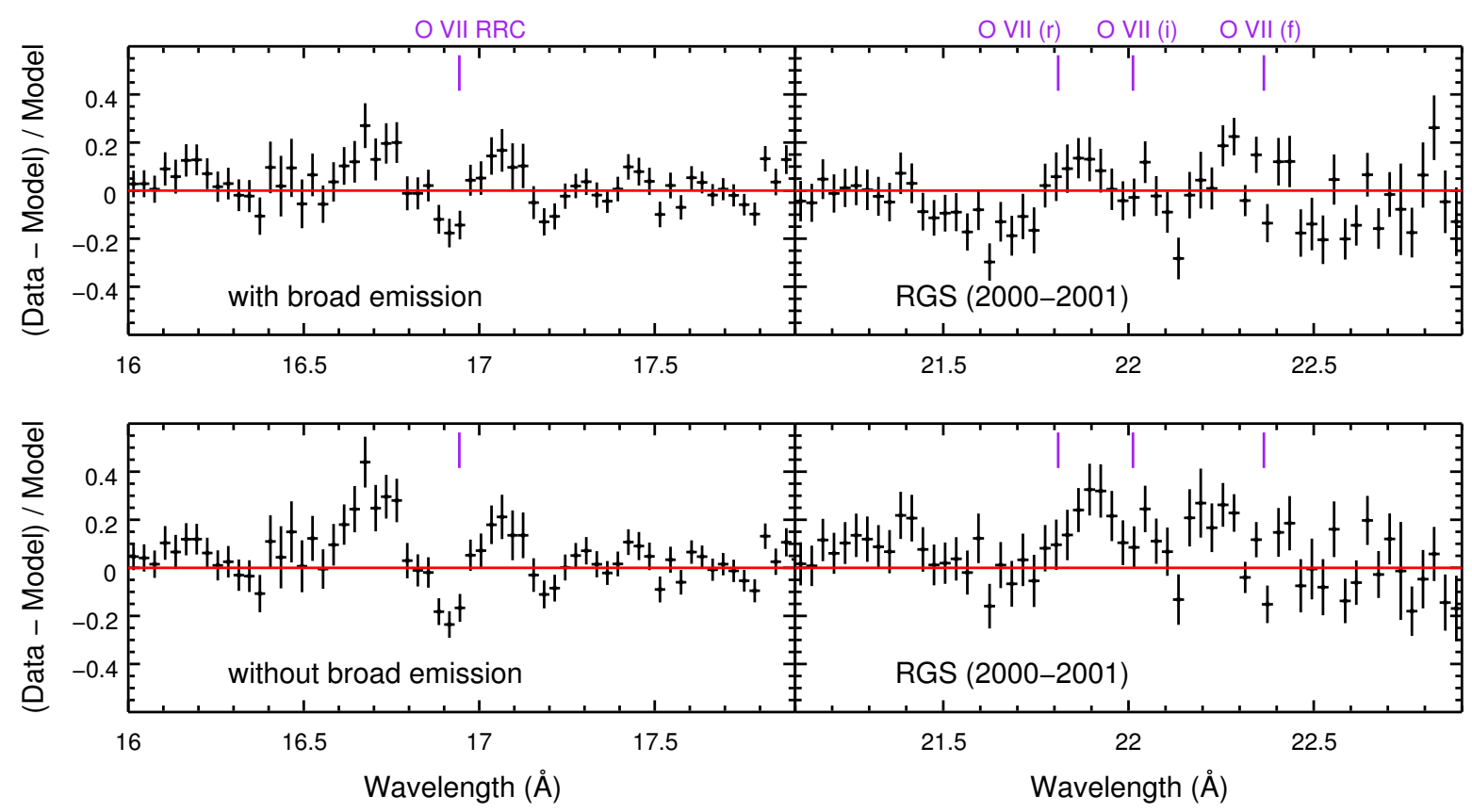

Fig. 6. Residuals of the best fit to the time-averaged RGS spectrum (in the observed frame) of NGC 3783 in 2000-2001 around the O vII RRC (left panels) and $\mathrm{O}$ viI He-like triplets (right panels). The upper and lower panels show residuals of the best fit with and without the broad $\left(F W H M \sim 21000 \mathrm{~km} \mathrm{~s}^{-1}\right)$ PION emission component (the third component in Table 4), respectively.

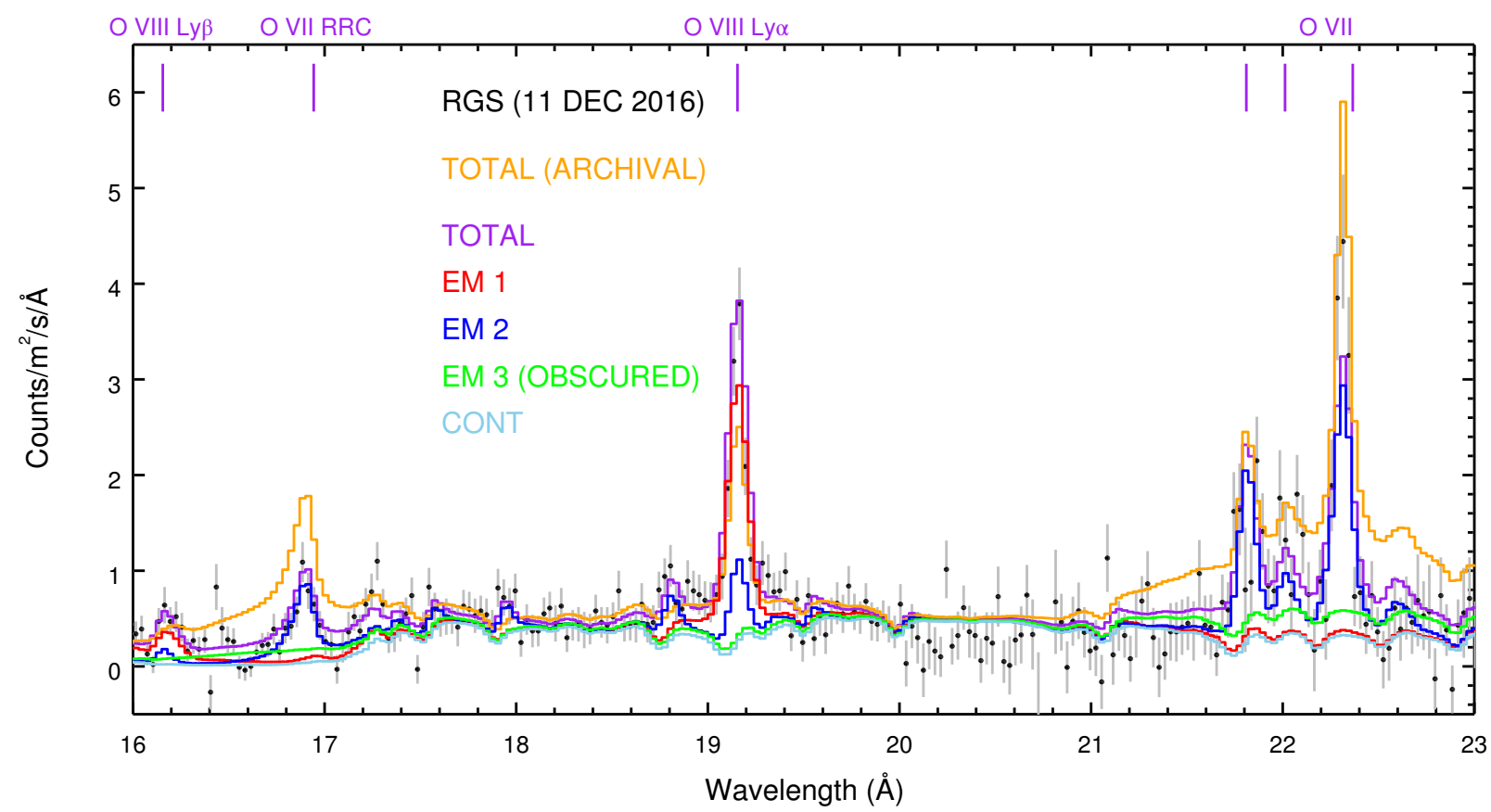

Fig. 7. Best fit to the 17-24 A RGS spectrum (in the observed frame) of NGC 3783 from 11 December 2016. The best-fit model with contributions from all emission components is shown in purple. Contributions from individual emission components are color-coded, with red and blue for narrow emission features and green for broad emission features. The orange solid line is a calculation using the best-fit parameters obtained from the time-averaged archival spectrum for all three emission components. Due to the obscuration effect, the broad emission component (EM 3) appears to be weaker in December 2016.

codes, and Mao et al. (2017) for a list of characteristic ground and metastable absorption lines.

\subsection{X-ray photoionized emitter}

In our PION modeling of the emission features (lines and $\mathrm{RRCs}$ ), we find that a broad emission component is required in addition to two narrow emission components. For the timeaveraged spectrum, the best fit with only narrow emission components yields a $C$-stat of 6301 with 2508 degrees of freedom, while the best fit with both broad and narrow emission components yields $\Delta C=-200$ at the price of three degrees of freedom. We tried values of the velocity broadening of $7000,8000,9000$, and $10000 \mathrm{~km} \mathrm{~s}^{-1}$; the respective best-fit 


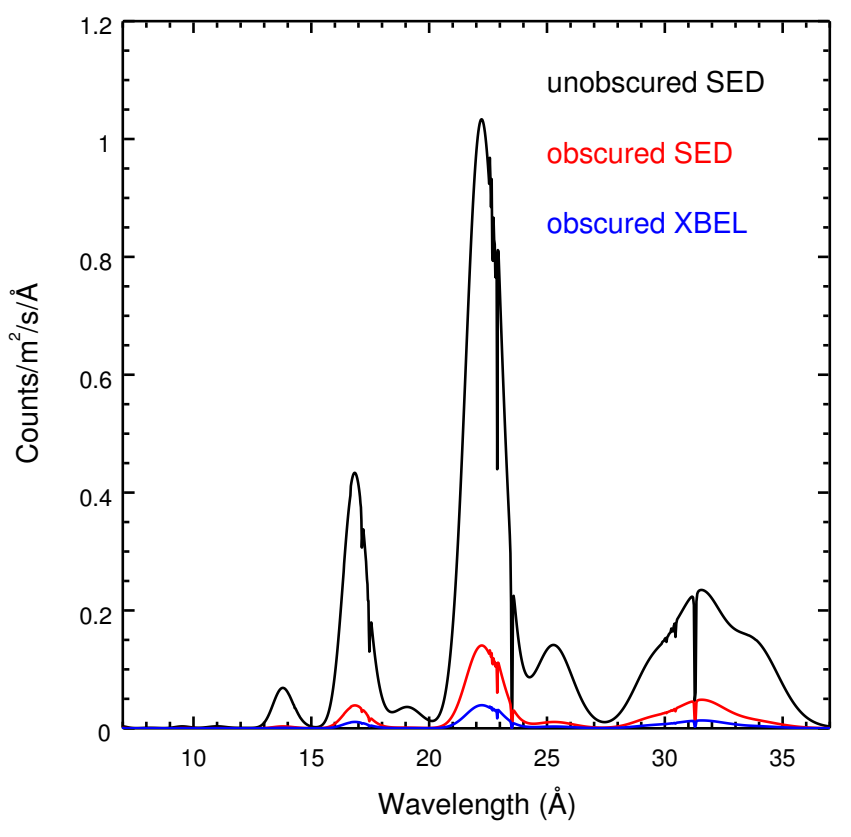

Fig. 8. Photoionization calculations of the X-ray broad emission lines (XBEL). In all three cases below, line profiles are corrected for the redshift and Galactic absorption of NGC 3783. The black solid curve corresponds to a case where the XBEL is directly exposed to the intrinsic SED of 11 December 2016. The red curve is for a case where the XBEL is exposed to the obscured SED of 11 December 2016. The blue curve is for a case where the XBEL is photoionized by the intrinsic SED of 11 December 2016 and further screened by the obscurer.

$C$-stats are 6108, 6098, 6092, and 6081. However, regardless of the presence of the broad emission component, there are still some structures in the residuals of the above two fits (Fig. 6). Future missions (e.g., Arcus, Smith et al. 2016) with adequate spectral resolution and a significantly larger photon collecting area are essential to verify the presence of such broad emission features in X-rays.

The broad emission component is obtained via convolving a narrow $\left(v_{\text {mic }}=100 \mathrm{~km} \mathrm{~s}^{-1}\right)$ PION component with a velocity broadening model (VGAU in SPEX) with a fixed $\sigma_{v}$, so that the broadening effect applies to both emission lines and RRCs. This broadening is probably due to the macroscopic motion of the emitter. Unfortunately, the velocity broadening in X-rays is a difficult parameter to determine. It cannot be too narrow, otherwise there is no significant improvement on the $C$-stat, nor can it be too broad, otherwise it is unclear whether we are fitting the broad emission line or a part of the complex continuum. Moreover, we are also limited by the calibration uncertainties of the effective area of the instrument, which can easily affect the value of the velocity broadening. At this stage, we tentatively fixed the value of $\sigma_{v}\left(=v_{\text {mac }}\right)$ to $9000 \mathrm{~km} \mathrm{~s}^{-1}$. This value is within the typical range of gas velocity $3000-10000 \mathrm{~km} \mathrm{~s}^{-1}$ (Blandford et al. 1990) in the broad-line region. For NGC 3783, the X-ray velocity broadening corresponds to a full width at half maximum $\left(F W H M=2.355 \sigma_{v}\right)$ of about $21000 \mathrm{~km} \mathrm{~s}^{-1}$, which is significantly larger than $F W H M(\mathrm{H} \beta) \sim 3000 \mathrm{~km} \mathrm{~s}^{-1}$ (Onken \& Peterson 2002) in the optical, but close to the width of the broadest component of Ly $\alpha$ in the UV, FWHM $18000 \mathrm{~km} \mathrm{~s}^{-1}$ (Kriss et al. 2019).

The broad and narrow emission model components derived from the 2000-2001 data do not match the data observed in December 2016 (orange line in Fig. 7). Narrow emission features might have varied slightly over the 15-year timescale (Sect. 4.4).
The broad emission component seems to be much weaker in December 2016 (Fig. 7). To explore possible explanations, we performed three sets of photoionization calculation of the X-ray broad emission lines, assuming the 2000-2001 parameters for X-ray broad emission lines. The first case is the simplest one where the XBEL is directly exposed to the intrinsic SED (the black curve in Fig. 8). In the presence of the obscurer, we need to consider two more cases. The XBEL might be exposed to the obscured SED (the red curve in Fig. 8), or the XBEL is photoionized by the intrinsic SED but further screened by the obscurer (the blue curve in Fig. 8). Of the three cases, the last one yields the weakest broad emission profiles. The second case yields a slight increase in $C$-stat $(\Delta C=+5)$ compared to the last one. Therefore, the apparent weakening observed in 2016 can be accounted for by applying the obscuration to the broad emission component (purple line in Fig. 7). However, we cannot strictly rule out the possibility that a fraction of the obscurer is co-existing or even closer to the black hole with respect to the $\mathrm{X}$-ray broad-line region. The parameters for the obscurer used here are the same as given in Table 1 of Mehdipour et al. (2017). The observed flux of the broad emission component around the O VII He-like triplets (21.0-23.6 $)$ decreases from $23 \times$ $10^{-17} \mathrm{~W} \mathrm{~m}^{-2}$ (2000-2001, i.e., "unobscured SED" in Figure 8) to $4 \times 10^{-17} \mathrm{~W} \mathrm{~m}^{-2}$ ("obscured SED"), and $1 \times 10^{-17} \mathrm{~W} \mathrm{~m}^{-2}$ (December 2016, i.e., "obscured XBEL"). The above interpretation supports the picture suggested by Mehdipour et al. (2017) that the obscurer is currently at the outer broad-line region of the AGN. Admittedly, if our assumption is not valid that the X-ray broad emission component varies significantly (column density, ionization parameter, and broadening), we can no longer break the degeneracy shown in Fig. 8.

The best fit to the RGS spectrum on 11 December 2016 is shown in Fig. 9, which is similar to that on 21 December 2016. The best-fit parameters of the X-ray photoionized emitter in 2000-2001 and December 2016 are listed in Table 4. While the emission measures $\left(E M=n_{\mathrm{e}} n_{\mathrm{H}} 4 \pi C_{\mathrm{em}} r^{2} N_{\mathrm{H}} / n_{\mathrm{H}}\right)$ of component 1 are consistent (at a $1 \sigma$ confidence level) between epochs, the emission covering factor $\left(C_{\mathrm{em}}\right)$, thus the emission measure of component 2 is an order of magnitude higher. If we fix $C_{\text {em }}($ EM 2) $=0.6$ when fitting the 11 December 2016 spectrum, the best-fit $C$-stat is 2406 , which is a worse fit $(\Delta C \sim+70)$ when compared to the best-fit $C$-stat in Table 2. Meanwhile, the ionization parameter of component 2 is a factor of two smaller in 2016; accordingly, the line emissivity is an order of magnitude lower in 2016. This might suggest an increase in the physical size of the emitter over 15 years.

Different values of the best-fit parameters of the narrow emission components in different epochs (Table 4) need to be interpreted with caution. Due to the lack of information, we assume the photoionization continuum to be the 2000-2001 AGN SED for all spectra in 2000-2001 and December 2016. Under this assumption, the ionization parameter $\xi=L /\left(n_{\mathrm{H}} r^{2}\right)$ should be the same, because the number density $\left(n_{\mathrm{H}}\right)$ and distance $(r)$ of the emission region are not expected to vary dramatically. The column density $\left(N_{\mathrm{H}}\right)$, microscopic turbulence velocity $\left(v_{\text {mic }}\right)$, and emission covering fraction $\left(C_{\mathrm{em}}\right)$ might not vary significantly as well. Deviation from the time-average photoionization continuum can be partly due to long-term variation in the intrinsic SED components and/or the obscuration events, which might be more frequent than previously thought for NGC 3783. Based on the hardness ratio of all Swift/XRT spectra in 2008-2017, obscuration events might occur about half of the time (Kaastra et al. 2018), which will affect the photoionization modeling of the X-ray narrow emission features. 


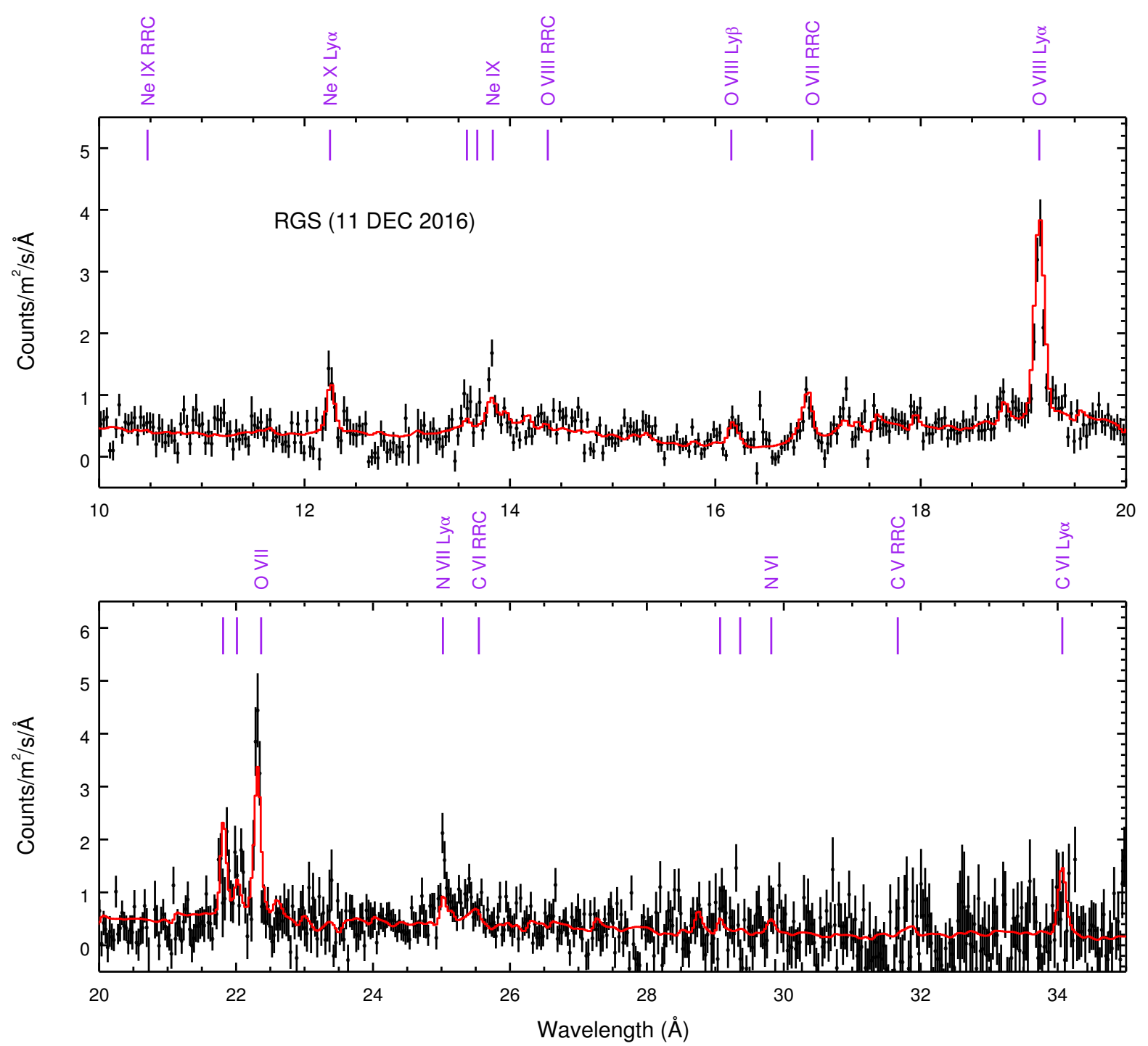

Fig. 9. Best fit to the RGS spectrum (in the observed frame) of NGC 3783 on 11 DEC 2016. Most of the prominent emission features are labeled.

Table 4. Best-fit results of the X-ray photoionized emitter in the time-averaged spectra of NGC 3783. The emission measure (E.M.) is calculated based on the best-fit parameters.

\begin{tabular}{cccccc}
\hline \hline Comp. & $\begin{array}{c}N_{\mathrm{H}} \\
10^{26} \mathrm{~m}^{-2}\end{array}$ & $\begin{array}{c}\log _{10}(\xi) \\
10^{-9} \mathrm{~W} \mathrm{~m}\end{array}$ & $\begin{array}{c}v_{\text {mic }} \\
\mathrm{km} \mathrm{s}^{-1}\end{array}$ & $\begin{array}{c}C_{\mathrm{em}} \\
\%\end{array}$ & $\begin{array}{c}\mathrm{EM} \\
10^{70} \mathrm{~m}^{-3}\end{array}$ \\
\hline \multicolumn{7}{c}{$2000-2001$} \\
\hline 1 & $60 \pm 37$ & $2.60 \pm 0.07$ & $600 \pm 100$ & $0.29 \pm 0.12$ & $0.5_{-0.4}^{+0.8}$ \\
3 & $5.2_{-2.3}^{+5.4}$ & $1.35 \pm 0.05$ & $140_{-100}^{+50}$ & $0.6_{-0.2}^{+0.4}$ & $1.5_{-1.0}^{+4.1}$ \\
& $28_{-5}^{+72}$ & $0.82 \pm 0.02$ & $100(\mathrm{f})$ & $0.3_{-0.2}^{+0.1}$ & $13_{-10}^{+53}$ \\
\hline \multicolumn{7}{c}{$11 \mathrm{DEC} 2016$} \\
\hline 1 & $25 \pm 6$ & $2.58 \pm 0.05$ & $590 \pm 90$ & $0.98 \pm 0.13$ & $1.0_{-0.4}^{+0.6}$ \\
3 & $3.0 \pm 0.7$ & $1.03 \pm 0.05$ & $350 \pm 70$ & $7.0 \pm 1.3$ & $30_{-13}^{+19}$ \\
\hline \multicolumn{7}{c}{$1.00(\mathrm{f})$} & $100(\mathrm{f})$ & $0.3(\mathrm{f})$ & 13 (f) \\
\hline 1 & $28(\mathrm{f})$ & $21 \mathrm{DEC} 2016$ \\
2 & $2.3 \pm 1.0$ & $1.03 \pm 0.05$ & $270 \pm 110$ & $7.3 \pm 2.4$ & $28_{-19}^{+32}$ \\
3 & 28 (f) & $1.06(\mathrm{f})$ & $100(\mathrm{f})$ & 0.3 (f) & 13 (f) \\
\hline \multicolumn{7}{c}{$2.58 \pm 0.05$} & $460 \pm 130$ & $0.7 \pm 0.3$ \\
\hline
\end{tabular}

Notes. Parameters followed by (f) are fixed in the fit. The 2000-2013 MEG (6-17 $\mathrm{A}$ ) data and the 2000-2001 RGS (7-37 $)$ data are fitted simultaneously (Sect. 3), but constraints are obtained mainly from the 2000-2001 RGS data since it covers all the emission features from Ne, O, $\mathrm{N}$, and C. Statistical uncertainties are quoted at the $68.3 \%$ confidence level. 


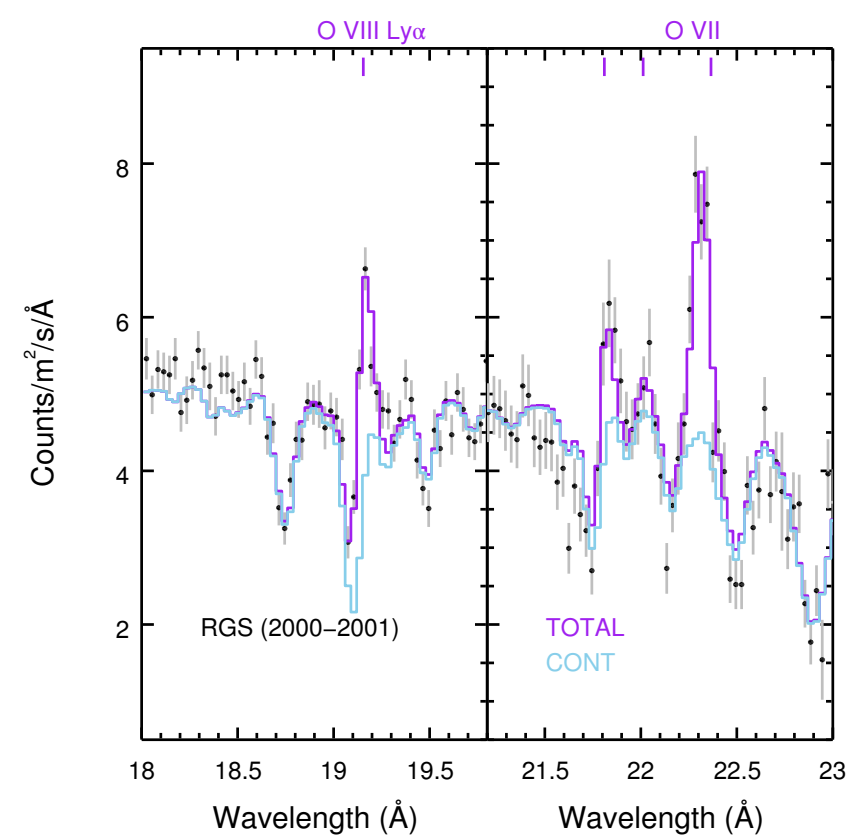

Fig. 10. Local fit to the observed $\mathrm{O}$ VIII $\operatorname{Ly} \alpha$ and $\mathrm{O}$ vII He-like triplets in the 2000-2001 time-averaged RGS spectrum of NGC 3783. The continuum model (CONT, light blue) is fixed to the best-fit global continuum, accounting for the foreground Galactic absorption and the warm absorber, and including the broad emission features.

Additionally, by comparing the best-fit results of the absorption (Table 3) and emission (Table 4) components, we find that emission component 1 and absorption component 3 have similar ionization parameters, yet the hydrogen column density, turbulence velocity, and outflow velocity are significantly different. The ionization parameter of emission component 2 has no counterpart in absorption components. This is similar to what we find in another Seyfert 1 galaxy NGC 5548 (Mao et al. 2018). It is possible that the X-ray emission and absorption components are not related, although we need distance (thus density) measurements for these components to verify this deduction.

\subsection{Variability of the $X$-ray emission features}

We check the variability of the most prominent narrow emission lines, the $\mathrm{O}$ VIII Ly $\alpha$ line and $\mathrm{O}$ VII He-like triplets, in the RGS spectra in 2000-2001 and December 2016. A phenomenological local fit is used in this exercise so that we are not confused by the unknown photoionization continuum effect on the photoionization modeling.

We fixed the continuum model to the best-fit global continuum accounting for the foreground Galactic absorption, the obscurer, the warm absorber, and included the broad emission features. Subsequently, the narrow emission lines are accounted for with Gaussian line profiles. The normalization and velocity broadening of the Gaussian profiles were left free to vary, except that we limited the velocity broadening to be no larger than $2000 \mathrm{~km} \mathrm{~s}^{-1}$ and coupled the broadening of the resonance and intercombination lines of the $\mathrm{O}$ vir He-like triplets. The line luminosity and velocity broadening are listed in Table 5 . The best-fit results of 2000-2001 and 11 December 2016 are plotted in Figs. 10 and 11.

These emission lines are consistent with each other at the $1 \sigma$ confidence level between the two observations in December 2016. When compared to 2000-2001, the O viI resonance and

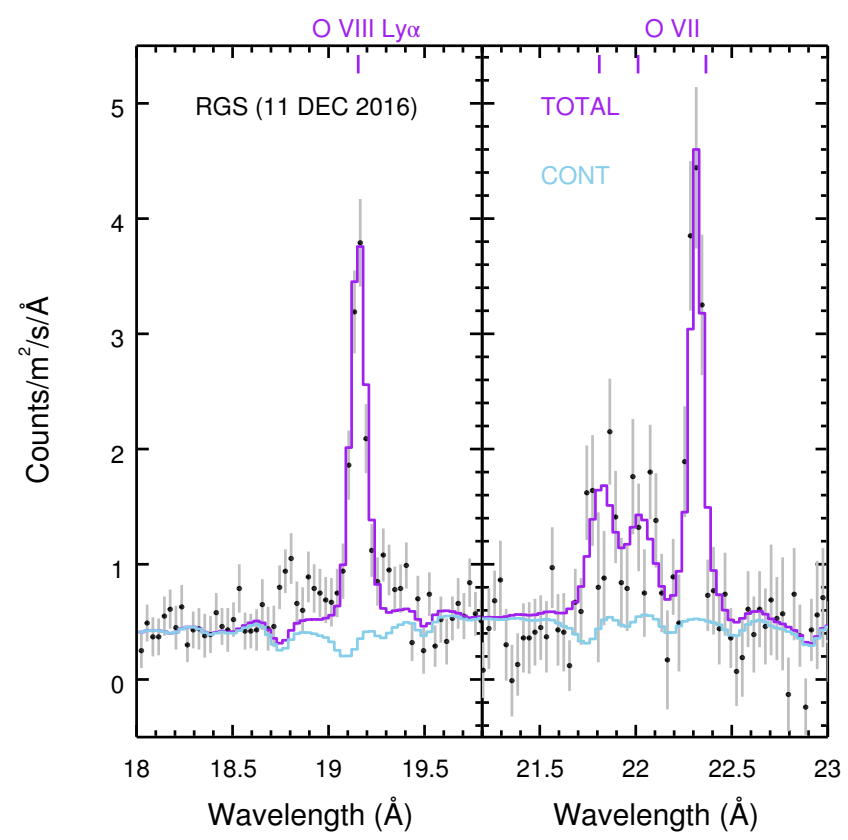

Fig. 11. Local fit to the observed O vIII Ly $\alpha$ (left panel) and O vII Helike triplets (right panel) in the RGS spectrum of NGC 3783 from 11 December 2016. The continuum model (CONT, light blue) is fixed to the best-fit global continuum, accounting for the foreground Galactic absorption, the obscurer, and the warm absorber, and including the obscured broad emission features.

forbidden lines remain constant in luminosity at the $1 \sigma$ confidence level, while the $\mathrm{O}$ vIII Ly $\alpha$ line and intercombination line of He-like O vil are marginally brighter in December 2016 at the $2 \sigma$ confidence level. In addition, the resonance and intercombination lines of O vil appear to be broader in December 2016, although the uncertainty is large. We note that the line luminosity depends on both the emission measure and line emissivity. While the emission measure of component 2 (Sect. 4.3) increases in 2016, the line emissivity decreases and leads to the comparable line luminosity between epochs.

We should point out that the residuals on both sides of the $\mathrm{O}$ vIII Ly $\alpha$ line in Fig. 11 are accounted for in the full plasma model fit shown in Fig. 7. A full plasma model includes many dielectric satellite lines, which are even weaker than the $\mathrm{O}$ vII $\mathrm{He} \beta$ line (at $18.63 \AA$ ).

Since the variability of the X-ray broad emission features cannot be checked directly, we turn to the very broad emission lines $\left(F W H M \gtrsim 10^{4} \mathrm{~km} \mathrm{~s}^{-1}\right)$ in the high-resolution UV spectra with HST in 2000-2001 and December 2016 (Kriss et al. 2019). No significant variation is found in the line flux of $\operatorname{Ly} \alpha$, Si Iv, $\mathrm{C}$ Iv, and He II between the two epochs. Hence, our previous assumption of a non-varying X-ray broad emission component is reasonable.

\subsection{Summary}

We analyzed the X-ray spectra of the Seyfert 1 galaxy NGC 3783 using both archival data in 2000-2013 and newly obtained data in December 2016. The intrinsic SED of the AGN, the obscuration, absorption, emission, and extinction effects are fitted simultaneously. In Fig. 12 we show a sketch of the relations among spectral components resulting from our modeling.

Along the line of sight toward the nucleus, prominent absorption line features due to the warm absorber were clearly 
Table 5. Best-fit results of the oxygen emission lines from RGS data in 2000-2001 and December 2016.

\begin{tabular}{|c|c|c|c|c|c|c|c|}
\hline \multirow[t]{2}{*}{ Ion } & \multirow{2}{*}{$\begin{array}{l}\text { Line } \\
\lambda(\AA)\end{array}$} & \multicolumn{2}{|c|}{ 2000-2001 } & \multicolumn{2}{|c|}{11 DEC 2016} & \multicolumn{2}{|c|}{21 DEC 2016} \\
\hline & & $L\left(10^{32} \mathrm{~W}\right)$ & $\sigma_{v}\left(\mathrm{~km} \mathrm{~s}^{-1}\right)$ & $L\left(10^{32} \mathrm{~W}\right)$ & $\sigma_{v}\left(\mathrm{~km} \mathrm{~s}^{-1}\right)$ & $L\left(10^{32} \mathrm{~W}\right)$ & $\sigma_{v}\left(\mathrm{~km} \mathrm{~s}^{-1}\right)$ \\
\hline $\mathrm{O}$ vIII Ly $\alpha$ & 18.97 & $13.4 \pm 0.9$ & $360 \pm 130$ & $17.8 \pm 1.1$ & $370 \pm 100$ & $19.8 \pm 1.1$ & $520 \pm 130$ \\
\hline O vII (r) & 21.60 & $6.6 \pm 1.4$ & $<280$ & $9.2 \pm 1.8$ & $740 \pm 190$ & $8.4 \pm 2.1$ & $<500$ \\
\hline O VII (i) & 21.81 & $1.3 \pm 1.3$ & $<280(\mathrm{c})$ & $6.0 \pm 1.6$ & $740(\mathrm{c})$ & $5.5 \pm 1.0$ & $<500$ (c) \\
\hline O vII (f) & 22.10 & $18.5 \pm 1.7$ & $310 \pm 100$ & $17.6 \pm 1.9$ & $<180$ & $17.3 \pm 3.1$ & $<300$ \\
\hline
\end{tabular}

Notes. Velocity broadening $\left(\sigma_{v}\right)$ of the resonance and intercombination lines of O vir are coupled (c) in the fit. Statistical uncertainties are quoted at the $68.3 \%$ confidence level.

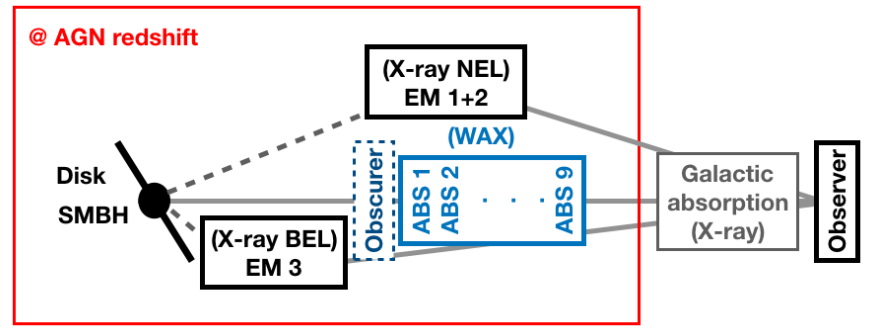

Fig. 12. Simplified view of the relations among spectral components observed in NGC 3783 in X-rays: warm absorber in X-rays (WAX), broad and narrow emission lines (BEL and NEL), and emission and absorption (EM and ABS, respectively). Three different light paths are shown here. The obscurer (dashed box) is not always present along the line of sight. Dashed lines indicate that these photons are not directly observable.

visible in the 2000-2013 X-ray spectra (e.g., Netzer et al. 2003; Krongold et al. 2003; Scott et al. 2014). Nine X-ray absorption components with different ionization parameters and kinematics are required in our photoionization modeling. On 11 and 21 December 2016, obscuration was seen in NGC 3783 (Mehdipour et al. 2017). The obscurer produces the heavy absorption of the soft X-ray continuum, as well as broad and blueshifted absorption lines in the UV spectra. It is very likely that there are more obscuration events in NGC 3783 (Markowitz et al. 2014; Kaastra et al. 2018).

The X-ray narrow emission components reprocess photons from the nucleus that are not directly observed (dashed lines in Fig. 12). Prominent emission features like the O vIII Ly $\alpha$ line and the He-like triplets of $\mathrm{O}_{\text {vII }}$ are visible whether the soft X-ray continuum is obscured or not. Weak emission features like the $\mathrm{O}$ VII RRC are detectable only when the continuum is obscured. Our photoionization modeling requires two components for the X-ray narrow emission features in NGC 3783. The two narrow emission components have different emission measures, ionization parameters, turbulence velocities, and emission covering factors. This is similar to our previous analysis for NGC 4151 (Seyfert 1.5, Armentrout et al. 2007), Mrk 335 (Seyfert 1, Longinotti et al. 2008), NGC 4051 (Seyfert 1, Nucita et al. 2010), NGC 424 (Seyfert 2, Marinucci et al. 2011), and NGC 5548 (Seyfert 1.5, Mao et al. 2018). On the other hand, Guainazzi et al. (2009) and Whewell et al. (2015) model the X-ray narrow emission features in NGC 1365 (Seyfert 1.8) and NGC 5548 (Seyfert 1.5), respectively, with only one photoionized component.

Our photoionization modeling (Table 4) finds that the ionization parameter of the X-ray narrow emission component 1 remains constant throughout 2000-2016, indicating that either this component has a distance of at least a few light-years or its density is very low. On the other hand, for the X-ray narrow emission component 2 , the ionization parameters are consistent between the two observations in December 2016 yet about a factor of two lower when compared to 2000-2001. If the variation is due to the change in the ionizing continuum, this suggests that component 2 is closer than component 1 , but its distance should be greater than 10 light-days.

The X-ray broad emission component also reprocesses photons from the nucleus. In this work, when the obscurer is present in NGC 3783, we demonstrate that it screens photons emitted from the X-ray broad emission component, which leads to the apparent weakening of the X-ray broad emission features in December 2016 (Sect. 4.3). The above interpretation supports the geometry proposed by Mehdipour et al. (2017) that the obscurer is located farther away than the $\mathrm{X}$-ray broad emission component, which is a few light-days from the nucleus (Peterson et al. 2004). Our photoionization modeling of the observed X-ray broad emission features is an ad hoc interpretation. There is evidence that the optical, UV, and X-ray broad emission lines originate from the same photoionized plasma (e.g., Costantini et al. 2016). Thus, the broadline region has a range of densities, ionization parameters, and kinematics. A dedicated analysis with the "locally optimally emitting cloud" approach (e.g., Costantini et al. 2007) or even sophisticated dynamic models (e.g., Pancoast et al. 2011) is required to interpret the optical to $\mathrm{X}$-ray broad emission lines in NGC 3783.

For simplicity, we assume that the X-ray broad and narrow emission components are not further absorbed by the warm absorber (Sect. 3), which might not be true. That is to say, the unabsorbed luminosity of the X-ray broad and narrow emission lines obtained here is only a lower limit. To properly account for the screening effects by the warm absorber, we need to know which warm absorber components are more distant than the $\mathrm{X}$-ray emission regions, and what are the covering factors for these warm absorber components with respect to the X-ray emission regions.

\section{Conclusions}

We focus on the photoionized emission features in the highresolution X-ray spectra of NGC 3783 obtained in December 2016 when the soft X-ray continuum was heavily obscured. We also analyze the archival time-averaged high-resolution X-ray spectrum in 2000-2001 to compare the photoionized emission features and study the warm absorber. The main results are summarized as follows:

1. Nine photoionization components with different ionization parameters and kinematics are required for the warm absorber; 
2. Two photoionization components are required for the X-ray narrow emission features, which are weakly varying over the past 15 years;

3. The presence of an X-ray broad emission component significantly improves the fit to the time-averaged spectrum in 2000-2001;

4. The X-ray broad emission features are much weaker in December 2016. This apparent weakening can be explained by the obscuration effect on the X-ray broad emission component.

Future missions like Athena/XIFU are needed to better constrain the properties of the warm absorber and X-ray emission features.

Acknowledgements. We thank the referee for the useful comments and suggestions. This work is based on observations obtained with XMM-Newton, an ESA science mission with instruments and contributions directly funded by ESA Member States and the USA (NASA). This research made use of data obtained with the NUSTAR mission, a project led by the California Institute of Technology (Caltech), managed by the Jet Propulsion Laboratory (JPL), and funded by NASA. This work made use of data supplied by the UK Swift Science Data Centre at the University of Leicester. SRON is supported financially by NWO, the Netherlands Organization for Scientific Research. This work was supported by NASA through a grant for HST program number 14481 from the Space Telescope Science Institute, which is operated by the Association of universities for Research in Astronomy, Incorporated, under NASA contract NAS526555. The research at the Technion is supported by the I-CORE program of the Planning and Budgeting Committee (grant number 1937/12). EC is partially supported by the NWO-Vidi grant number 633.042.525. CP acknowledges funding through ERC Advanced Grant number 340442. EB is grateful for funding from the European Union's Horizon 2020 research and innovation program under the Marie Sklodowska-Curie grant agreement no. 655324. SB acknowledges financial support from ASI under grant ASI-INAF I/037/12/0, and from the agreement ASI-INAF n. 2017-14-H.O. GP acknowledges support from the Bundesministerium für Wirtschaft und Technologie/Deutsches Zentrum für Luftund Raumfahrt (BMWI/DLR, FKZ 50 OR 1408) and the Max Planck Society. BDM acknowledges support by the Polish National Science Center gran Polonez 2016/21/P/ST9/04025. POP acknowledges support from the CNES and French PNHE. LDG acknowledges support from the Swiss National Science Foundation.

\section{References}

Armentrout, B. K., Kraemer, S. B., \& Turner, T. J. 2007, ApJ, 665, 237 Behar, E., Rasmussen, A. P., Blustin, A. J., et al. 2003, ApJ, 598, 232 Bentz, M. C., Denney, K. D., Grier, C. J., et al. 2013, ApJ, 767, 149 Bianchi, S., Guainazzi, M., \& Chiaberge, M. 2006, A\&A, 448, 499 Bennert, N., Jungwiert, B., Komossa, S., Haas, M., \& Chini, R. 2006a, A\&A, 459, 55

Bennert, N., Jungwiert, B., Komossa, S., Haas, M., \& Chini, R. 2006b, A\&A, 456, 953

Beuchert, T., Markowitz, A. G., Dauser, T., et al. 2017, A\&A, 603, A50

Blandford, R. D., Netzer, H., Woltjer, L., Courvoisier, T. J. L., \& Mayor, M. 1990, Active Galactic Nuclei (Berlin Heidelberg: Springer-Verlag), 97

Blustin, A. J., Branduardi-Raymont, G., Behar, E., et al. 2002, A\&A, 392, 453

Canizares, C. R., Davis, J. E., Dewey, D., et al. 2005, PASP, 117, 1144

Chelouche, D., \& Netzer, H. 2005, ApJ, 625, 95

Costantini, E., Kaastra, J. S., Arav, N., et al. 2007, A\&A, 461, 121

Costantini, E., Kriss, G., Kaastra, J. S., et al. 2016, A\&A, 595, A106

den Herder, J. W., Brinkman, A. C., Kahn, S. M., et al. 2001, A\&A, 365, L7

Denney, K. D., De Rosa, G., Croxall, K., et al. 2014, ApJ, 796, 134

De Rosa, A., Piro, L., Fiore, F., et al. 2002, A\&A, 387, 838

Detmers, R. G., Kaastra, J. S., \& McHardy, I. M. 2009, A\&A, 504, 409
Ebrero, J., Kriss, G. A., Kaastra, J. S., \& Ely, J. C. 2016, A\&A, 586, A72 Elvis, M., Wilkes, B. J., McDowell, J. C., et al. 1994, ApJS, 95, 1

Fu, X.-D., Zhang, S.-N., Sun, W., Niu, S., \& Ji, L. 2017, Res. Astron. Astrophys., 17, 095

Fukumura, K., Kazanas, D., Shrader, C., et al. 2018, ApJ, 853, 40

Goosmann, R. W., Holczer, T., Mouchet, M., et al. 2016, A\&A, 589, A76

Green, J. C., Froning, C. S., Osterman, S., et al. 2012, ApJ, 744, 60

Guainazzi, M., Risaliti, G., Nucita, A., et al. 2009, A\&A, 505, 589

Hönig, S. F., Kishimoto, M., Tristram, K. R. W., et al. 2013, ApJ, 771, 87

Kaastra, J. S., Mewe, R., Liedahl, D. A., Komossa, S., \& Brinkman, A. C. 2000, A\&A, 354, L83

Kaastra, J. S., Detmers, R. G., Mehdipour, M., et al. 2012, A\&A, 539, A117

Kaastra, J. S., Kriss, G. A., Cappi, M., et al. 2014, Science, 345, 64

Kaastra, J. S., Mehdipour, M., Behar, E., et al. 2018, A\&A, 619, A112

Kaspi, S., Brandt, W. N., Netzer, H., et al. 2000, ApJ, 535, L17

Kaspi, S., Brandt, W. N., Netzer, H., et al. 2001, ApJ, 554, 216

Kaspi, S., Brandt, W. N., George, I. M., et al. 2002, ApJ, 574, 643

Kraemer, S. B., Crenshaw, D. M., \& Gabel, J. R. 2001, ApJ, 557, 30

Kriss, G. A., Mehdipour, M., Kaastra, J. S., et al. 2019, A\&A, 621, A12

Krongold, Y., Nicastro, F., Brickhouse, N. S., et al. 2003, ApJ, 597, 832

Krolik, J. H. 1999, Active Galactic Nuclei : from the Central Black Hole to the Galactic Environment (Princeton, NJ: Princeton University Press)

Landt, H., Ward, M. J., Steenbrugge, K. C., \& Ferland, G. J. 2015, MNRAS, 454, 3688

Liedahl, D. A., \& Paerels, F. 1996, ApJ, 468, L33

Lodders, K., \& Palme, H. 2009, Meteorit. Planet. Sci. Suppl., 72, 5154

Longinotti, A. L., Nucita, A., Santos-Lleo, M., \& Guainazzi, M. 2008, A\&A 484,311

Longinotti, A. L., Costantini, E., Petrucci, P. O., et al. 2010, A\&A, 510, A92

Magdziarz, P., \& Zdziarski, A. A. 1995, MNRAS, 273, 837

Mao, J., Kaastra, J. S., Mehdipour, M., et al. 2017, A\&A, 607, A100

Mao, J., Kaastra, J. S., Mehdipour, M., et al. 2018, A\&A, 612, A18

Marinucci, A., Bianchi, S., Matt, G., et al. 2011, A\&A, 526, A36

Markowitz, A. G., Krumpe, M., \& Nikutta, R. 2014, MNRAS, 439, 1403

Mason, K. O., Breeveld, A., Much, R., et al. 2001, A\&A, 365, L36

Mehdipour, M., Kaastra, J. S., Kriss, G. A., et al. 2015, A\&A, 575, A22

Mehdipour, M., Kaastra, J. S., \& Kallman, T. 2016, A\&A, 596, A65

Mehdipour, M., Kaastra, J. S., Kriss, G. A., et al. 2017, A\&A, 607, A28

Netzer, H., Kaspi, S., Behar, E., et al. 2003, ApJ, 599, 933

Nicastro, F., Fiore, F., Perola, G. C., \& Elvis, M. 1999, ApJ, 512, 184

Nucita, A. A., Guainazzi, M., Longinotti, A. L., et al. 2010, A\&A, 515, A47

Ogle, P. M., Mason, K. O., Page, M. J., et al. 2004, ApJ, 606, 151

Onken, C. A., \& Peterson, B. M. 2002, ApJ, 572, 746

Pancoast, A., Brewer, B. J., \& Treu, T. 2011, ApJ, 730, 139

Parker, M. L., Alston, W. N., Buisson, D. J. K., et al. 2017, MNRAS, 469, 1553

Peterson, B. M. 1997, An Introduction to Active Galactic Nuclei (Cambridge, NY: Cambridge University Press), 238

Peterson, B. M., Ferrarese, L., Gilbert, K. M., et al. 2004, ApJ, 613, 682

Peterson, B. M., Denney, K. D., De Rosa, G., et al. 2013, ApJ, 779, 109

Ramírez, J. M., Bautista, M., \& Kallman, T. 2005, ApJ, 627, 166

Rivers, E., Risaliti, G., Walton, D. J., et al. 2015, ApJ, 804, 107

Scott, A. E., Brandt, W. N., Behar, E., et al. 2014, ApJ, 797, 105

Silva, C. V., Uttley, P., \& Costantini, E. 2016, A\&A, 596, A79

Smith, R. K., Abraham, M. H., Allured, R., et al. 2016, Proc. SPIE, 9905 99054M

Steenbrugge, K. C., Fenovčík, M., Kaastra, J. S., Costantini, E., \& Verbunt, F. 2009, A\&A, 496, 107

Strüder, L., Briel, U., Dennerl, K., et al. 2001, A\&A, 365, L18

Theureau, G., Bottinelli, L., Coudreau-Durand, N., et al. 1998, A\&AS, 130, 333

Titarchuk, L. 1994, ApJ, 434, 570

Turner, T. J., Reeves, J. N., Braito, V., et al. 2018, MNRAS, 481, 2470

Whewell, M., Branduardi-Raymont, G., Kaastra, J. S., et al. 2015, A\&A, 581, A79

Zycki, P. T., Done, C., \& Smith, D. A. 1999, MNRAS, 305, 231 


\section{Appendix A: Component relation in fitting the spectra using SPEX}

The emission and absorption components of our model for the X-ray spectrum of NGC 3783 have a complex spatial relationship that is important for the construction our model in SPEX. Figure 12 gives a visual representation of our model. The intrinsic SED originates in the accretion disk surrounding the supermassive black hole (SMBH). This is the innermost component of the model. This innermost emission region is absorbed by both the obscurer, when it is present (model component 2), and the Xray warm absorber (WAX). The next emission layer is the X-ray BEL (part of model component 4). This is also absorbed by the obscurer when it is present. The outermost emission component is the X-ray NEL (part of model component 4), which may be cospatial with the WAX, but we presume that it is not absorbed by the WAX. The final component in our model is Galactic absorption (model component 7), which affects all other components originating in NGC 3783.

The intrinsic SED of NGC 3783 consists of a Comptonized disk component (COMT in SPEX), the power-law component (POW), and a neutral reflection component (REFL). These are additive components. An exponential cutoff is applied to the high- and low-energy end of the power-law component. This is realized via the ETAU model in SPEX (see also Chapter 7 of the SPEX cookbook), which provides a simple transmission $T(E)=\exp \left(-\tau_{0} E^{a}\right)$, where $\tau_{0}$ is the optical depth at $1 \mathrm{keV}$. For the high-energy cutoff, we set $a=1$ and $\tau_{0}=1 / 340$, which corresponds to a cutoff energy at $340 \mathrm{keV}$ (De Rosa et al. 2002). For the low-energy cutoff, we set $a=-1$ and $\tau_{0}=T_{\text {seed, }}$, which corresponds to a cutoff at the seed photon temperature $\left(T_{\text {seed }}\right)$ of the Comptonized disk component.

The warm absorber is modeled with nine PION absorption components, which are multiplicative components. The most highly ionized component (index 1) is assumed to be the closest to the nucleus, while the least ionized component (index 9) is assumed to be the furthest. In the absence of the obscurer, the first PION absorption component is directly exposed to the AGN SED. Ionizing photons received by the second PION absorption is the AGN SED screened by the first PION absorption component. The rest is done in the same manner. In the presence of the obscurer, photons from the nucleus are first screened by the obscurer then modified by PION absorption components.

The PION emission components (for the X-ray photoionized emitter) are both multiplicative and additive. This is because they reprocess ionizing photons which are not directly observed (dashed lines in Fig. 12) and emit photons which are directly observed. Accordingly, the ionizing continuum is first multiplied by the PION emission components (as multiplicative components) then followed by a ETAU component with $\tau_{0}=1000$ and $a=0$ so that this ionizing continuum is not present in the spectrum. As additive components, the PION emission components are multiplied by the redshift of the AGN and the Galactic absorption in X-rays.

We provide a rough estimate of the distance of the X-ray photoionized emitter in the following. To estimate the distance of the narrow- and broad-line gas we need the mass of the supermassive black hole, the geometry factor, and the broadening of the line due to Keplerian motion (e.g., Eq. (1) of Peterson et al. 2004). The geometry factor is usually assumed to be 5.5 (Peterson et al. 2004), but the broadening of the line due to Keplerian motion is unknown in our case. We do have measurement on the line broadening, but for the narrow emission line components we attribute the broadening to microscopic turbulence. We note that turbulence affects the broadening of the line and also the strength of the line. We attempted to add a macroscopic motion to the narrow-line gas, but we cannot obtain a meaningful constraint $\left(v_{\text {mac }} \ll v_{\text {mic }}\right)$. That is to say, we only have the upper limit on the line broadening due to Keplerian motion, which yields the lower limit of the distance $(>1 \mathrm{pc})$ of the narrow-line gas.

For the broad-line gas, the X-ray FWHM is broader than that of the optical, indicating that the X-ray emitting gas is closer to the black hole than the optical emitting gas. The distance of the optical BLR ranges from 1.4 light-days to 10.2 light-days (Peterson et al. 2004). Thus, we have a reasonable upper limit of the distance $(<1.4$ light-days) of the $\mathrm{X}$-ray broad-line gas.

The distance of the obscurer is about 10 light-days, according to Mehdipour et al. (2017). Thus, the obscurer is farther away than the X-ray BLR but closer than the X-ray NLR. 